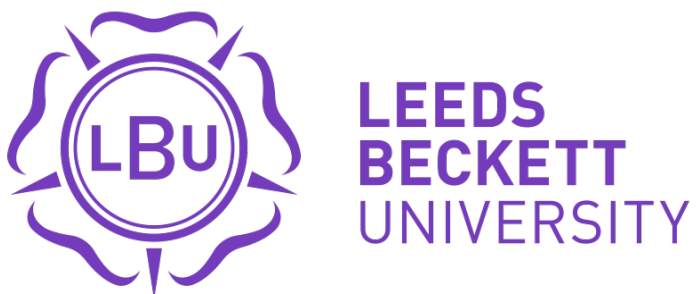

Citation:

Perepelkin, NV and Argatov, II and Borodich, FM (2019) Evaluation of elastic and adhesive properties of solids by depth-sensing indentation. The Journal of Adhesion. pp. 1-42. ISSN 0021-8464 DOI: https://doi.org/10.1080/00218464.2019.1686981

Link to Leeds Beckett Repository record:

https://eprints.leedsbeckett.ac.uk/id/eprint/6686/

Document Version:

Article (Accepted Version)

This is an Accepted Manuscript of an article published by Taylor \& Francis in The Journal of Adhesion on 10 Nov 2019, available online: http://www.tandfonline.com/10.1080/00218464.2019.1686981

The aim of the Leeds Beckett Repository is to provide open access to our research, as required by funder policies and permitted by publishers and copyright law.

The Leeds Beckett repository holds a wide range of publications, each of which has been checked for copyright and the relevant embargo period has been applied by the Research Services team.

We operate on a standard take-down policy. If you are the author or publisher of an output and you would like it removed from the repository, please contact us and we will investigate on a case-by-case basis.

Each thesis in the repository has been cleared where necessary by the author for third party copyright. If you would like a thesis to be removed from the repository or believe there is an issue with copyright, please contact us on openaccess@leedsbeckett.ac.uk and we will investigate on a case-by-case basis. 


\section{Evaluation of elastic and adhesive properties of solids by depth-sensing indentation}

Nikolay V. Perepelkin ${ }^{a, c 1}$, Ivan I. Argatov $^{b}$, Feodor M. Borodich ${ }^{a}$

a School of Engineering, Cardiff University, Cardiff, CF24 3AA, UK

${ }^{b}$ Department of Materials Science and Applied Mathematics, Malmö University, SE-20506 Malmö, Sweden

${ }^{c}$ Department of Applied Mathematics, National Technical University "Kharkiv Polytechnic Institute", Kharkiv, 61002, Ukraine

${ }^{1}$ Corresponding author. E-mail address: PerepelkinM@cardiff.ac.uk 


\section{Evaluation of elastic and adhesive properties of solids by depth-sensing indentation}

To describe properly interactions between contacting solids at micro/nanometre scales, one needs to know both adhesive and mechanical properties of the solids. Borodich and Galanov have introduced an effective method (the BG method) for identifying both characteristics from a single experiment on depth-sensing indentation by a spherical indenter using optimal fitting of the experimental data. Unlike traditional indentation techniques involving sharp indenters, the Borodich-Galanov methodology intrinsically takes adhesion into account. It is essentially a nondestructive approach. These features extend the scope of the method to important applications beyond the capabilities of conventional indentation. The scope of the original BG method was limited to the classic JKR and DMT theories. Recently, this restriction has been overcome by introducing the extended BG (eBG) method, where a new objective functional based on the concept of orthogonal distance curve fitting has been introduced. In the present work, questions related to theoretical development of the eBG method are discussed. Using the data for elastic bulk samples, it is shown that the eBG method is at least as good as the original BG method. It is shown that the eBG can be applied to adhesive indentation of coated, multilayered, functionally-graded media.

Keywords: the BG method, depth sensing indentation (DSI), mechanical properties of adhesives, analytical models, non-destructive testing, coatings.

\section{Introduction}

To solve many problems of modern nanomechanics, one needs to consider not only the mechanical interactions between solids but also to take into account effects caused by various physical and chemical effects of interacting surfaces. In particular, one needs to consider adhesion between contacting solids. The effects of adhesion become increasingly significant as the contact size decreases [1] and hence, evaluation of adhesive characteristics of solids is extremely important for describing their interactions at micro- or nano-scales. Subjects and specimens in the modern Materials Science and Nanotechnology are often of very small dimensions, e.g. membranes of biological cells, many surface coatings and layers deposited on solids are very thin. Clearly, one cannot employ the conventional tests such as the uni-axial tensile tests of macroscale samples [2] or the tape peeling tests [3] for evaluation the mechanical and adhesive properties of such tiny solids. Therefore, various indentation tests of materials are often employed. The depth-sensing indentation (DSI) tests became especially popular in the last decades for studying properties of materials of very small volumes. The experimental data of a DSI test is presented as arrays of measurements of the force $P$ applied to the indenter and the corresponding displacements $\delta$ of the indenter. These measurements can be presented as the force-displacement curve or the $P-\delta$ curve [4]. If one associates the $P-\delta$ curve with a mathematical model of the indentation process, then the material properties may be evaluated by solving an inverse problem of fitting the model to the experimental data.

Usually adhesive and mechanical characteristics of contacting solids are evaluated employing two independent and rather different indentation tests: (i) DSI of sharp pyramidal indenters for extraction of the effective contact modulus $E^{*}$ from the 
unloading branch of the $P-\delta$ curve; and (ii) extraction of work of adhesion $w$ from direct measurements of the pull-off force (the adherence force $P_{a d h}$ which is assumed to be negative) of a spherical indenter from the material sample and sometimes using in the calculation few other points of the indentation $P-\delta$ curve $[5,6]$. Both of the above techniques have several drawbacks that will be briefly discussed in the next section (see also discussion in $[7,8]$ ). Hence, for many modern materials, both characteristics, especially the work of adhesion, are not well known quantities [9].

After analysis of the experimental techniques based on the use of pyramidal indenters, Chaudhri and Lim [10] suggested to determine elastic constants of materials from the Hertz formulae using the initial elastic stage of the force-displacement curves when the material samples are loaded by spherical indenters. However, the $P-\delta$ curves of such tests may be greatly influenced by adhesion of materials. In turn, Borodich and Galanov [11] introduced an effective method (the BG method) for identifying both elastic and adhesive characteristics from a single DSI experiment employing optimal fitting of the experimental curve by an appropriate wellestablished model of adhesive contact of spheres.

The evaluation of adhesive properties of contacting materials is possible by solving an inverse problem using the ideal theoretical $P-\delta$ curve corresponding to a chosen mathematical theory of the indentation process. In other words, if a mathematical model of the indenter-specimen interaction is specified, then one can fit experimental data points with the expected theoretical $P-\delta$ curve via adjustment of model parameters related to unknown material properties. However, each experimental measurement is affected by variation of properties of the material tested and other sources of experimental noise. That is why, in contrast to other methods of mechanics of materials, the BG method uses the entire set of data points of unloading branch of the force-displacement curve rather than some specific points on it. The BG method allows the researchers to extract simultaneously both the adhesive and elastic characteristics from the experimental DSI diagrams by employing a nondirect approach. The BG method is based on a simple idea that at low loads the $P-\delta$ curves noticeably reflect not only elastic properties but also adhesive properties of the contact. Therefore, one can simultaneously identify from experiments both elastic characteristics of contacting materials and characteristics of molecular adhesion. It was observed that the compressive part of the adhesive $P-\delta$ diagram is much less sensitive to roughness than its tensile part. The BG approach suggests to fit the experimental data on the stable stages of the indentation diagrams by a theoretical curve of the appropriate model of adhesive contact, i.e. by solving an inverse problem. The experimental data fitting is supposed to be done through adjustment of the values of characteristic scales of the contact problem (the quantities needed to re-write the theoretical force-displacement dependency in a dimensionless form). Once the values of the characteristic scales have been extracted from the reliable data, mechanical and adhesive parameters can be calculated. In particular, the values of the pull-off force, the minimum value of the displacement, the work of adhesion, and the reduced elastic modulus of the materials. It was shown by Borodich et al. [12-14] that the BG method is robust at least within the range of applicability of the JKR theory.

It is noteworthy, that the scope of the $B G$ method extends to a number of important cases in which the use of common indentation techniques (e.g. the one by Oliver and Pharr [15]) is undesired or impossible: 
(i) it can be used for determination of adhesive properties in the first instance, or whenever adhesion during DSI cannot be neglected (i.e. micro/nanoindentation of soft solids);

(ii) it is espesially suitable for the use together with the Colloidal Probe technique [16-18], which uses cantilever-mounted spherical particles as probes;

(iii) it is a non-destructive technique. This provides benefits in terms of: (a) the ability to test living biological samples (e.g. cell membranes); (b) the ability to do repetitive tests at exactly the same location (e.g. to do statistical averaging of the results, or to investigate surface physics under changing environment conditions); (c) non-destructive control of the quality and properties of protective coatings [19], including those working in aggressive environment.

Recently, Perepelkin et al [20] have announced an extension of the BG method. The main difference between the original BG method and its extended version is that the latter is based not on the known versions of the JKR or DMT theories that are strictly speaking applicable only to elastic half-spaces, but on the use of parametrically defined force-displacement curves. It is shown below that the extended BG (eBG) method can be applied not only to elastic half-spaces but also to thin and thick layered systems. Another specific feature of the eBG method is the use of the orthogonal distance fitting approach. This leads to a different objective functional of data fitting. The paper [20] should be considered as an announcement of the eBG method and a proof-of-concept because the eBG method was presented "as is", without thorough justification of the particular workflow of the method and the new objective functional used. The work [20] has presented a direct experimental validation of the new approach, which constitutes the main part of that paper. In particular, it has been shown there, that the values of the elastic contact modulus extracted from indentation tests by the extended BG method are in a good agreement with the values obtained by standard macroscale tensile tests.

In contrast, the present work is focused on theoretical development of the eBG method. It demonstrates two different implementations of this method as a framework that consists of (a) experimental part (DSI by spherical indenters), and (b) data processing part (orthogonal data fitting for a parametrically defined forcedisplacement curve). One of these implementations was earlier used in [20], but practically no detail was given. Here we show exactly how and why the proposed modifications of the original BG method follow from the use of the orthogonal distance fitting concept and the attempt to avoid unnecessary large scale optimization. Apart from different implementation of the eBG method, the following questions are presented below: (i) the discussion and simulations related to convergence and accuracy of the new approach, (ii) matters related to the determination of the unknown coordinate origin for the measured indenter displacement are discussed in detail, (iii) generalized variance as the measure of goodness of fitting related to the settings of the fitting algorithm is introduced. As a demonstration of capabilities of the eBG method, it is shown that it can be applied to adhesive indentation of coated, multilayered, or functionally-graded media. All these additional details are of interest for scientific community and enable the reader to understand better the scope of the eBG method, performance-related matters in terms of speed and accuracy, and the complete workflow of the method.

The paper is organized as follows. Section 2 contains brief discussion regarding commonly used DSI techniques, and the importance of accounting adhesion phenom- 
ena in DSI. Further, in section 3, the specific features of the original BG method are described. Then the theoretical aspects of the extended BG (eBG) method are discussed.

Two implementations of the extended BG method are given. In the first version, the theoretical force-displacement curve given by two parametric functions is fitted directly to the experimental data in the framework of orthogonal distance fitting (ODF) approach. This approach emerges naturally as the result of finding the curve parameter values corresponding to a selected data points located off the theoretical curve, i.e. the projections of the data points onto an ideal theoretical curve are considered. Due to the non-linear nature and high computational cost of ODF, the ODF algorithms for explicit functions began to emerge only in 1980s [21] while the algorithms for parametric and implicit functions and surfaces have been the matter of recent studies (e.g. [22-26]). In the first variant of the extended BG method, the data points projections and the characteristic scales of the BG method are determined iteratively from a series of interleaved minimizations of the objective functional. The problem dimension in the ODF approach is rather high because both the characteristic scales of the BG method and projection points are unknown.

The second version of the eBG method implies that noisy experimental data is initially smoothed (pre-fitted) with some auxiliary curve formulated as an explicit function. Then, the theoretical DSI curve is "fitted" to the auxiliary one. The purpose of doing so is twofold. Firstly, this approach allows one to use some advanced fitting techniques since the auxiliary curve is supposed to have very simple mathematical form. This auxiliary curve also acts as a filter greatly reducing the amount of measurement noise present in the experimental data. Secondly, the objective functional can now be formulated in such a way that there is no need in finding projections of the data points onto theoretical curve since the corresponding values of the parameter along the curve are excluded from the functional. This modification essentially reduces the problem's dimension and computation time while maintaining good precision. This allows one to use complex mathematical models of adhesive contact to effectively identify material properties from DSI results. In the present study the asymptotic model by Argatov et al. [27] is used for numerical testing of the proposed eBG method. It is shown here that the eBG can be applied to adhesive indentation of coated, multilayered, or functionally-graded media; as an example, a problem of adhesive indentation of an elastic coating is described in detail.

In Section 4 accuracy, robustness and convergence speed of the proposed approaches are examined. The results of a number of numerical simulations and the results of processing a real experimental data are presented. Some specific aspect related to the adhesive DSI tests are also discussed, e.g. problems related to unknown origin of coordinates related to the $P-\delta$ curve. The algorithm of extraction of the sought values from real experimental data are described in detail. Using the data for elastic bulk samples, it is shown that the eBG method is at least as good as the original BG method.

\section{DSI as an adhesive contact problem}

To explain the advantages of the BG and eBG methods, we need first to briefly discuss the common usage and the respective drawbacks of the traditional DSI techniques, as well as the influence of adhesion in DSI experiments. 
First DSI techniques were described in late 1960s [4]. Shortly, Bulychev et al. [28] would realize that the slope of the $P-\delta$ curve could be linked to the value of the effective (reduced) contact elastic modulus $E^{*}$ of the pair "indenter-specimen". As long as DSI techniques were originally applied using sharp pyramidal indenters, like the Berkovich indenter [29], Bulychev et al. suggested to use a known exact relation for the slope $S$ of the $P-\delta$ curve $S=d P / d \delta=2 E^{*} a$ for axisymmetric indenters in an altered way [28] (the so-called BASh relation):

$$
S=d P / d \delta=2 E^{*} \sqrt{A / \pi}
$$

In fact, the contact radius $a$ was replaced with the expression $\sqrt{A / \pi}$, where $A$ is the current contact area, thus making the formula not exact, but rather an approximate relation. Here $E^{*}$ denotes the effective (reduced) contact elastic modulus: $\left(E^{*}\right)^{-1}=$ $\left(1-\nu^{2}\right) / E+\left(1-\nu_{i}^{2}\right) / E_{i}$, where the elastic moduli and Poisson's ratios of the specimen and the indenter are denoted as $E, \nu$, and $E_{i}$ and $\nu_{i}$ respectively (if the indenter is rigid, i.e. $E_{i}=\infty$, then $\left.E^{*}=E /\left(1-\nu^{2}\right)\right)$.

The BASh relation (1) was then applied to the case of pyramidal indenters and has become the corner stone of modern instrumented indentation. However, there are various complications in clearly interpreting the indentation results, e.g., a complication arises from the "pile-up" or "sink-in" of the material around the indenter (see, e.g. [30]). Indeed, one would need to solve a very complex contact problem to calculate the current contact area $A$ analytically. Therefore, various semi-empirical procedures for estimations of $A$ have been introduced, for instance, the one described by Oliver and Pharr [15].

Despite its popularity and being de facto an industrial standard [31], the Oliver and Pharr (OP) approach has such intrinsic drawbacks as destructive nature and inability to account adhesion. We have to underline that the derivation of the BASh formula (1) and the OP approach are based on the assumptions of nonadhesive Hertz contact theory and even if the area of the current contact region is calculated exactly, they cannot be used for determination of the adhesive properties of contacting materials. As it is mentioned in the Introduction, there is a number of important cases in which the OP approach is undesired, or cannot be used properly. See also discussions in $[7,10,32,33]$.

Inevitable presence of adhesion in every DSI experiment has two aspects. The first aspect of the presence of adhesion in DSI is that DSI can be specifically used to determine adhesive properties of materials [5, 6, 34-36].

In spite of considerable efforts applied to studies of adhesive contact problems, only the classic theories of adhesive frictionless contact are well established, including the JKR [37], DMT [38] and Maugis (see e.g. [39]) theories. These classic theories allow explicit representation of the $P-\delta$ curve only if an elastic half-space is loaded by a spherical indenter. The latter fact allows the identification of the work of adhesion $w$ based on the use of direct methods [5,6]. The discussed methods vary depending on the device and the theory employed. Apparently, the most popular approach for estimations of the work of adhesion is based on the direct experimental measurements of the adherence force $P_{a d h}$ between a sphere of radius $R$ and an elastic half-space in combination with the use of the JKR model. Assuming that the surfaces of the tested sample and the probe are ideally smooth, and applying directly the JKR model, one obtains 


$$
w=-\frac{2}{3} \frac{P_{a d h}}{\pi R} .
$$

However, the values of $P_{a d h}$ obtained by direct measurements have usually poor reproducibility because the tensile (adhesive) part of the $P-\delta$ diagram may be greatly influenced by surface roughness of the specimen. Grierson et al. [40] argue that pull-off measurements can be influenced by a number of factors (especially at nano-scale) such as the roughness of contacting surfaces (see also [9]) and surface chemistry, wear of DSI probe and chemical modification of its surface (in case of DSI by means of atomic force microscopy (AFM)) etc. Therefore, the tensile part of DSI load-displacement diagram can be considered unstable and less trustworthy.

Hence, one needs to have a large number of experimental measurements to estimate $w$ properly using Eq. (2) or using a similar method based on direct measurements of the force of adhesion. In addition, techniques, which have been developed for determination of work of adhesion from direct measurements of the adhesive force, are valid mostly for experimental conditions when the JKR theory is applicable [39].

The second reason to study DSI as an adhesive contact problem is that adhesion can distort the results of a DSI experiment, if the experimental approach does not take adhesion into account [11]. This point is supported by other authors, e.g. Kohn and Ebenstein [41] stated that "although adhesion leads to overestimation of the modulus of compliant samples when analyzing nanoindentation data using traditional analysis techniques, most studies of biomaterials have ignored its effects". The same reference suggests that "compliant, hydrated materials exhibit very different mechanical behaviors relative to mineralized tissues, and require significant modifications to traditional indentation methods to measure accurate modulus values".

As long as the base indentation method is based on a non-adhesive contact theory, introduction of some extra correction steps are needed [41,42]. However, if adhesion cannot be neglected, the entire methodology should be rather built around an approach that takes adhesion into account intrinsically. This was the main motivation that lead to the creation of the BG method described in the following subsection.

\section{The BG method and its extensions}

It is important to mention that, broadly speaking, we see the BG method not just as a fitting procedure, but rather as a framework that combines various techniques in a single procedure, similarly to the well-known instrumental indentation method introduced by Oliver and Pharr [15,31]. The first step of both the BG and extended BG (eBG) methods, presented here, is indentation by a spherical indenter (although the spherical shape is not necessary for the methods, this shape provides a number of advantages that are discussed above, such as non-destructive nature, etc.) The second step consists of preliminary choice of the theory of adhesive contact to be used (JKR, Maugis, DMT, etc.), and fitting the corresponding mathematical model of the indentation process to the experimental data, thus evaluating unknown material properties. The third step is the validation of the initial choice of the theory of adhesive contact used in step two. It is done via calculation of the Tabor-Muller pa- 
rameter using the estimated material properties. The Tabor-Muller parameter separates the domains of applicability of different theories of adhesive contact. Hence, if the calculated value does not correspond to the initially used theory of adhesive contact, another theory and a different mathematical model should be chosen and the fitting procedure should be repeated.

In the present section the developments related to fitting experimental data in the framework of the BG method are discussed, whereas section 4.3 demonstrates what the complete workflow of the BG/eBG method may look like, including the validation of the initial choice of the theory of adhesive contact used for computations.

\subsection{The BG method}

First we consider the original BG method as the ground for our subsequent considerations. The BG method was developed for simultaneous evaluation of elastic and adhesive properties of bulk isotropic material samples in the framework of either Johnson-Kendall-Roberts (JKR) or Deriaguin-Muller-Toporov (DMT) theories of adhesive contact. In fact, it is a model-based method, i.e. a pre-built mathematical model of the indentation process in the form of $P-\delta$ curve is used where an expected relation between the measured force and displacement values can be written as the following function $F$ :

$$
F\left(\frac{P}{P_{c}}, \frac{\delta}{\delta_{c}}\right)=0
$$

The exact form of this relation depends on the assumed physical model of adhesive contact (JKR, DMT, etc). Note that equation (3) represents the $P-\delta$ curve using dimensionless force $P / P_{c}$ and dimensionless displacement $\delta / \delta_{c}$. The parameters $P_{c}>0$ and $\delta_{c}>0$ are so-called characteristic (or scaling) parameters of the problem. They are connected to unknown material properties via dependencies which are known a priori in the framework of the chosen contact model.

For example, for the JKR theory of adhesive contact in the case of a spherical indenter of radius $R$ equation (3) can be written in the form of piece-wise dependency [39]:

$$
\begin{cases}(3 \chi-1)\left(\frac{1+\chi}{9}\right)^{\frac{1}{3}}-\frac{\delta}{\delta_{c}}=0 & \text { for } \chi \geq 0, \frac{\delta}{\delta_{c}} \geq-3^{-2 / 3} \\ (3 \chi+1)\left(\frac{1-\chi}{9}\right)^{\frac{1}{3}}-\frac{\delta}{\delta_{c}}=0 & \text { for } \frac{2}{3} \geq \chi \geq 0,-3^{-2 / 3}>\frac{\delta}{\delta_{c}} \geq-1\end{cases}
$$

where $\chi=\sqrt{1+\frac{P}{P_{c}}}$. The characteristic parameters $P_{c}$ and $\delta_{c}$ are connected to the reduced Young's modulus of material $E^{*}$ and work of adhesion $w$ by means of the following formulae:

$$
P_{c}=\frac{3}{2} \pi w R, \quad \delta_{c}=\frac{3}{4}\left(\frac{\pi^{2} w^{2} R}{E^{* 2}}\right)^{1 / 3} .
$$

The above scaling parameters were also used to construct dimensional form of the JKR force-displacement relation in the papers by Borodich et al. [11-14,20]. 
In the case of the JKR theory the parameters $P_{c}$ and $\delta_{c}$ have clear physical meanings, e.g. $P_{c}$ is the maximum possible absolute value of the pull-off force during unloading phase of indentation. If another theory of adhesive contact is used, then one needs to write other equations instead of (4) and therefore, $P_{c}$ and $\delta_{c}$ are just scaling parameters that have a different physical meaning than discussed above.

Let us consider the outcomes of $N$ measurements $\left(\delta_{i}, P_{i}\right), i=1 \ldots N$ of the indentation depth $\delta_{i}$ and indentation force $P_{i}$ during DSI. If the measurements are error-free, then each data point $\left(\delta_{i}, P_{i}\right)$ belongs to the theoretical $P-\delta$ curve. Therefore, one has a system of $N$ exact equalities

$$
\left\{F\left(\frac{P_{i}}{P_{c}}, \frac{\delta_{i}}{\delta_{c}}\right)=0, \quad i=1 \ldots N .\right.
$$

Hence, any two of equations (6) could be picked and solved to find the unknowns $P_{c}$ and $\delta_{c}$, and we can use three equations if we add to the sought values the unknown shift $\delta_{s}$ of coordinate origin of the $P-\delta$ curve.

In fact, the real experimental data contains measurement errors and therefore, all the expressions in (6) cannot be true simultaneously. This means that (6) is an overdetermined system of non-linear equations with respect to $P_{c}$ and $\delta_{c}$ that has no solution in classical sense. However, one can find some optimal values of $P_{c}$ and $\delta_{c}$ by means of minimization of a certain measure of the total "error" produced in (6). Such measure can be a norm of the set of residuals in (6). If one selects the mean square residual as the measure of the total "error" in (6), then the optimal values of the characteristic parameters $P_{c}^{*}, \delta_{c}^{*}$ can be found as the result of minimization of the objective functional of the problem $\Phi\left(P_{c}, \delta_{c}\right)$ :

$$
\left\{P_{c}^{*}, \delta_{c}^{*}\right\}=\arg \min \Phi\left(P_{c}, \delta_{c}\right),
$$

where

$$
\Phi\left(P_{c}, \delta_{c}\right)=\sum_{i=1}^{N}\left[F\left(\frac{P_{i}}{P_{c}}, \frac{\delta_{i}}{\delta_{c}}\right)\right]^{2} .
$$

As the result, the optimal values of the characteristic parameters allow one to best fit the theoretical force-displacement curve to the experimental data in the sense of the metric (8). In comparison to other fitting approaches, the BG method (7)-(8) has its own distinctive features:

(i) The metric (8) differs from the one normally introduced in least-squares curve fitting (LSF), therefore producing different optimum point. In fact, the LSF approach minimizes the fitting residual with respect to only one variable. Therefore, it is implicitly supposed that the second variable is error-free which is not the case in any real experiment. The original BG method does not have the above assumption.

(ii) The method uses fitting curve written in dimensionless form which allows to treat quantities of different orders of magnitude in the same way.

(iii) The fitting process is performed through adjusting characteristic scales $P_{c}$ and $\delta_{c}$ but not the material properties.

In addition, the method successfully allows to estimate $E^{*}$ and $w$ using only compressive part of the load-displacement data if needed, hence, using only stable measurements $[12,13]$.

In the general case of models of contact adhesive interactions, the external load and the indenter displacements may be presented only through parametric functions with the contact radius as the parameter [8]. In particular, such parametric 
dependencies may arise as the result of extension of a force-displacement relationship from non-adhesive case to adhesive one in the framework of the JKR theory. As an important example, one can consider mathematical models of indentation of layered and coated medium. Even in the simplest case of indentation of a thin layer bonded to a rigid support the adhesive force-displacement relationship cannot be reduced to explicit form unless indenter has a spherical shape [43]. Analytical JKR force-displacement curves for the frictionless contact of an indenter and a coated elastic medium may have very complex representation which cannot be reduced to explicit form for any indenter shape $[27,44]$. The same is valid for the cases in which other contact theories of adhesive contact are used to study coated, multilayered, or functionally-gradient medium (e.g. the Maugis theory [45, 46], or the double-Hertz theory [47]). In addition, it is fundamentally impossible to reduce semi-analytical models which contain correction functions computed numerically by means of the Finite Elements Method to explicit form [48-50]. The above examples are presented in more detail in Appendix A.

Nevertheless, all the above mentioned mathematical models can be utilized for simultaneous determination of elastic and adhesive properties of elastic structures using the approach presented in this work. Apparently, equation (3) is not the only possible representation of theoretical force-displacement curve. In some important cases, e.g. described above, the force-displacement curve is represented as a parametric function, and it is rather impossible to exactly convert it into the form (3).

Thus, in its initial formulation the BG method cannot utilize mathematical models which describe force-displacement curve as a parametric function. In the following subsections we address this issue by extending the concept and applicability scope of the BG method. The extended BG method allows one to use forcedisplacement relations formulated in the parametric form, so that the DSI plots are represented by parametric curves. The eBG method can be applied to a wide number of important complexly formulated problems of Materials Science which cannot be exactly reduced to explicit or implicit functions, and this is demonstrated in the present work by means of both numerical simulations and processing real experimental data.

\subsection{The BG method in the context of orthogonal data fitting approach}

Let us re-formulate the concept of the original BG method using different definition of the fitting residual. The purpose of such re-formulation is to be able to use the theoretical force-displacement curve in the form of a parametric function:

$$
\left\{\begin{array}{l}
\frac{\delta}{\delta_{c}}=f_{1}\left(\bar{a}, \delta_{c}, P_{c}\right), \\
\frac{P}{P_{c}}=f_{2}\left(\bar{a}, \delta_{c}, P_{c}\right) .
\end{array}\right.
$$

Here, $\bar{a}$ is the dimensionless parameter, which may have physical interpretation, e.g. dimensionless contact radius. However, this meaning is not mandatory.

If the force-displacement curve is represented in the form (9), one can develop a measure of fitting residual that has clear geometrical meaning. Consider an $i$-th data point in dimensionless force-displacement coordinates (using some arbitrary 
characteristic scales $P_{c}$ and $\delta_{c}$ ). If the measurements are absolutely exact, then the data point belongs to the theoretical force-displacement curve and has a corresponding value of the parameter $\bar{a}=\bar{a}_{i}$ (Figure 1). It this ideal situation the equations (9) are fully satisfied.

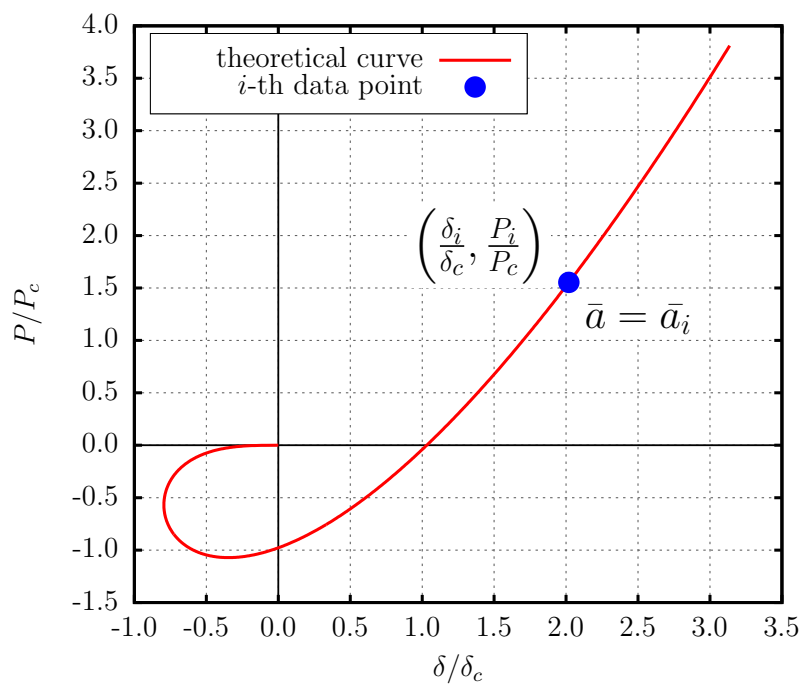

Figure 1: Ideal situation: a data point belongs to the theoretical force-displacement curve (9) in dimensionless force-displacement coordinates.

Let us introduce the following expression as the measure of misfit of the $i$-th data point and the theoretical curve:

$$
\boldsymbol{\Phi}_{i}\left(P_{c}, \delta_{c}, \bar{a}_{i}\right)=\left(\frac{\delta_{i}}{\delta_{c}}-f_{1}\left(\bar{a}_{i}, \delta_{c}, P_{c}\right)\right)^{2}+\left(\frac{P_{i}}{P_{c}}-f_{2}\left(\bar{a}_{i}, \delta_{c}, P_{c}\right)\right)^{2} .
$$

Clearly, $\boldsymbol{\Phi}_{i}=0$ in the above ideal situation due to (9).

Now consider a real measurement. Due to measurement errors the $i$-th data point does not belong to the theoretical curve. In fact, one has two points: the first one is the data point which is located off-curve and the second one is the point on the curve that corresponds to the value of the parameter $\bar{a}=\bar{a}_{i}$ (Figure 2a). In this case the value of $\boldsymbol{\Phi}_{i}$ becomes square of the distance between the two points. If $\boldsymbol{\Phi}_{i}$ is then minimized with respect to $\bar{a}_{i}$, the value of $\boldsymbol{\Phi}_{i}$ becomes square of the shortest possible distance between the two points. That is, the point corresponding to $\bar{a}=\bar{a}_{i}$ is the projection of the $i$-th data point onto the theoretical curve and the value of $\boldsymbol{\Phi}_{i}$ is square of the orthogonal distance from the data point to the theoretical curve (Figure 2b).

Now, recalling that the overall number of measurements is $N$ one can introduce the following metric to define the total fitting residual of $N$ data points:

$\boldsymbol{\Phi}\left(\delta_{c}, P_{c}, \bar{a}_{1}, . . \bar{a}_{N}\right)=\sum_{i=1}^{N} \boldsymbol{\Phi}_{i}=\sum_{i=1}^{N}\left[\left(\frac{\delta_{i}}{\delta_{c}}-f_{1}\left(\bar{a}_{i}, \delta_{c}, P_{c}\right)\right)^{2}+\left(\frac{P_{i}}{P_{c}}-f_{2}\left(\bar{a}_{i}, \delta_{c}, P_{c}\right)\right)^{2}\right]$.

When minimized with respect to all the variables $\left\{\delta_{c}, P_{c}, \bar{a}_{1}, . . \bar{a}_{N}\right\}$, this functional allows one to find the load-displacement curve which best fits experimental data in terms of minimal averaged value of squared orthogonal distance from the data 


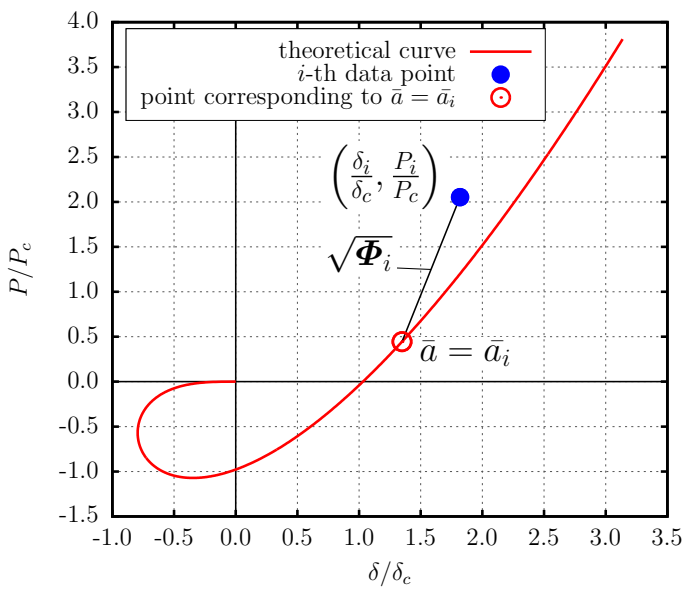

(a)

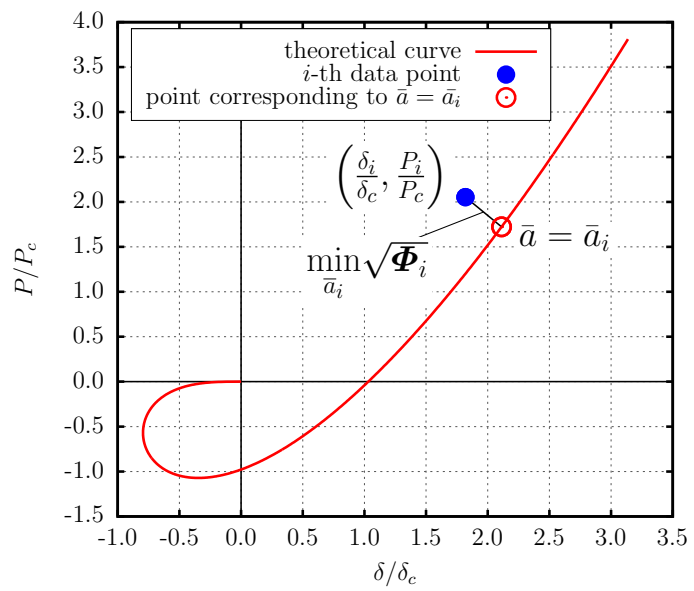

(b)

Figure 2: Relative position of the $i$-th data point (solid circle) and the point on the theoretical curve that corresponds to the value of the parameter $\bar{a}=\bar{a}_{i}$ (circle) in real situation. (a) The value of $\bar{a}_{i}$ is arbitrary. (b) The value of $\bar{a}_{i}$ is optimal.

points to the curve (so-called orthogonal distance curve fitting (ODF) approach [21, 25]). This approach is useful when both abscissas and ordinates of the data points are subject to measurement errors. The optimal values of the characteristic parameters $P_{c}^{*}, \delta_{c}^{*}$ can be found as the result of minimization of the objective functional in the form (11) with respect to all of its variables:

$$
\left\{P_{c}^{*}, \delta_{c}^{*}\right\}=\arg \min _{\delta_{c}, P_{c}, \bar{a}_{i}} \boldsymbol{\Phi}\left(\delta_{c}, P_{c}, \bar{a}_{1}, . . \bar{a}_{N}\right) .
$$

Thus, the BG method has been re-formulated in the framework of the ODF approach and the new objective functional (11) has been introduced.

Note that there exist other works on the determination of material properties by means of direct fitting the theoretical DSI curve to experimental data, in particular, obtained by atomic force microscopy (AFM) technique. For instance, Domke and Radmacher [51], Dimitriadis et al. [52] determined the apparent Young's modulus of thin organic films by means of fitting AFM data to a Hertz-like analytical model. Recently, a number of authors [53-55] determined elastic and adhesive properties of living cells using mostly the JKR contact model; Notbohm et al. [56] identified properties of a silicone rubber by means of DSI with small sphere by AFM technique. Lin et al. $[57,58]$ developed algorithms of the evaluation of material properties using the Hertz non-adhesive and the Pietrement-Troyon adhesive models of frictionless contact. However, it should be emphasized that except the latter two works in most cases a little or no information is provided about fitting methods and procedures. Neither mathematical aspects of the employed fitting approaches (contrary to the paper [11]), nor readiness to utilize highly noisy experimental data are discussed. This aspect is very important as demonstrated in the present paper. Also, in all the above mentioned papers the mathematical models of contact interaction $(P-\delta$ curves) are supposed to be explicit functions which can be achieved only in simplest cases. Thus, the eBG method has an advantage because it can be applied to rather complex problems (e.g. contact with coated medium).

The main issue of the optimization problem (11)-(12) is the large number of problem variables which is equal to $N+2:\left\{\delta_{c}, P_{c}, \bar{a}_{1}, . . \bar{a}_{N}\right\}$. Hence, the ODF prob- 
lems are known to be computationally intensive and complex [21,25]. Although the simultaneous minimization of (12) with respect to all its variables is possible, is not desirable because normally $N$ is large (up to a few thousands).

In the subsequent subsections we discuss two approaches that address the above issue. In the former one, all the $N+2$ variables of (12) are kept in place, however the minimization process is performed for different variables separately. In the latter one, the ODF approach is used for preliminary fitting the experimental data with an auxiliary non-parametric curve. The theoretical curve is then fitted to the auxiliary one using the objective functional in the form of integral. As the result, there is no need in evaluation of parameter values $\bar{a}_{i}$ and hence, the number of problem variables is greatly reduced.

\subsection{Direct data fitting}

In the present subsection we consider the problem of search for the optimal values of $P_{c}$ and $\delta_{c}$ in the form (11)-(12). In fact, the theoretical curve is fitted to the data points directly without any auxiliary steps.

Despite the fact that the simultaneous minimization of the functional (11) with respect to all $N+2$ unknowns is possible, it can be noted that minimization with respect to $\bar{a}_{i}$ can be done separately for each $\bar{a}_{i}$ when $\delta_{c}, P_{c}$ are fixed since $\frac{\partial \Phi}{\partial \bar{a}_{i}}$ contains only $\bar{a}_{i}$ :

$$
\frac{\partial \boldsymbol{\Phi}}{\partial \bar{a}_{i}}=-2\left[\left(\frac{\delta_{i}}{\delta_{c}}-f_{1}\left(\bar{a}_{i}, \delta_{c}, P_{c}\right)\right) \frac{\partial f_{1}}{\partial \bar{a}_{i}}+\left(\frac{P_{i}}{P_{c}}-f_{2}\left(\bar{a}_{i}, \delta_{c}, P_{c}\right)\right) \frac{\partial f_{2}}{\partial \bar{a}_{i}}\right]
$$

Due to (13) the optimization problem can be solved by means of two-level iterative minimization process that is also referred to as the variable-separation method $[25,26])$ :

$$
\min \boldsymbol{\Phi}=\min _{\delta_{c}, P_{c}} \min _{\bar{a}_{1} . . \bar{a}_{N}} \boldsymbol{\Phi} .
$$

The flow of this two-level interleaving minimization process can be expressed as the following algorithm.

1. Assign initial values (guess) to $P_{c}$ and $\delta_{c}$.

2. Update the values of $\bar{a}_{i}$.

(a) Lock the assigned values of $P_{c}$ and $\delta_{c}$.

(b) For each $\bar{a}_{i}$ minimize the objective functional with respect to $\bar{a}_{i}$ while keeping the rest of the variables constant.

(c) Update the values of $\bar{a}_{i}$ with the found optimal ones.

3. Update the values of $P_{c}$ and $\delta_{c}$.

(a) Lock the assigned values of all $\bar{a}_{i}$.

(b) Minimize the objective functional with respect to $P_{c}$ and $\delta_{c}$ while keeping the rest of the variables constant.

(c) Update the values of $P_{c}$ and $\delta_{c}$ with the found optimal ones. 
4. If the global optimality condition is not met, then go to step 2. Otherwise, end cycle.

5. The current values of $P_{c}$ and $\delta_{c}$ become the globally optimal ones $P_{c}^{*}$ and $\delta_{c}^{*}$.

At any given step, the number of variables engaged in minimization process does dot exceed two. This reduces greatly computational complexity. On the other hand, this approach is similar to coordinate descent optimization method and is expected to be rather slowly convergent. For this reason, an approach that uses not only the idea of orthogonal distance fitting but also reduces greatly the number of variables and computational time is considered in the next subsection.

\subsection{The extended BG method with preliminary fitting}

As actual expressions (9) may be very complex, multiple re-evaluation of values $\bar{a}_{i}$ may become very time-consuming. On the other hand, the researcher is not interested in obtaining these values since only $\delta_{c}, P_{c}$ are the matter of interest. The approach presented in the present subsection allows one to exclude evaluation of $\bar{a}_{i}$ during the identification of $P_{c}$ and $\delta_{c}$.

Clearly, the aim of fitting data points with the theoretical curve is not the fitting itself but rather finding the unknown material properties evaluated from optimal values of $P_{c}$ and $\delta_{c}$ with reasonable accuracy using reasonable amount of computation time. Hence, some additional steps may be introduced into fitting the theoretical curve to the data points to speed up computations.

Here we present the concept of preliminary fitting (pre-fitting) of the experimental data. The main idea is to fit experimental data with some auxiliary curve $P=\Psi(\delta)$ (Figure 3) on the first stage of evaluation of $\delta_{c}, P_{c}$. Mathematical expression of this new curve must be simple enough to make fitting the data quick and easy process. Due to its simplicity, the auxiliary curve may be constructed using some advanced fitting techniques like ODF. Alternatively, the pre-fitting curve may be the result of some kind of post-processing/filtering of the experimental data.

On the second stage the values of $P_{c}$ and $\delta_{c}$ are adjusted in such way that the theoretical curve becomes closest to the auxiliary one in certain sense. It can be done without calculation of individual values of $\bar{a}_{i}$ even if the theoretical loaddisplacement curve is represented in parametric form. This speeds up computation drastically while maintaining good accuracy of the final result. The objective functional of the BG method can be constructed as follows.

Consider the theoretical force-displacement curve in the form

$$
\left\{\begin{array}{c}
\delta=\delta_{c} f_{1}\left(\bar{a}, \delta_{c}, P_{c}\right), \\
P=P_{c} f_{2}\left(\bar{a}, \delta_{c}, P_{c}\right) .
\end{array}\right.
$$

Let the experimental data be pre-fitted with an auxiliary curve $P=\Psi(\delta)$ on the interval $\left[\delta_{\min }, \delta_{\max }\right]$ where $\delta_{\min }=\min \left(\delta_{i}\right), \delta_{\max }=\max \left(\delta_{i}\right), i=1 \ldots N$. Then in order to approximate the initial data well the norm of difference of the two functions (the theoretical $P-\delta$ curve and the auxiliary one) must be minimized which leads to minimization of the following functional: 


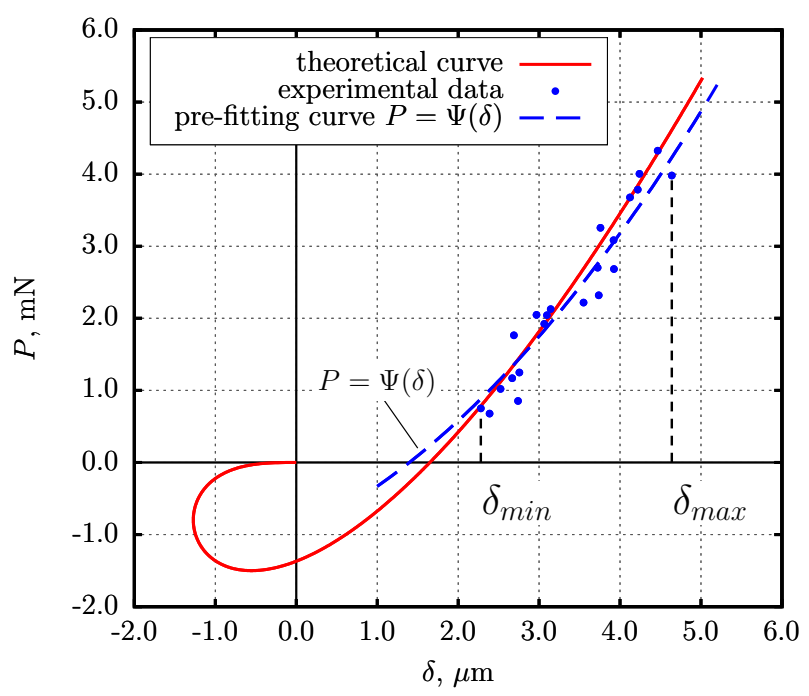

Figure 3: Pre-fitting the experimental data with an auxiliary curve $\Psi(\delta)$.

$$
\hat{\boldsymbol{\Phi}}\left(P_{c}, \delta_{c}\right)=\int_{\delta_{\min }}^{\delta_{\max }}[P(\delta)-\Psi(\delta)]^{2} d \delta
$$

Here $P=P(\delta)$ is the theoretical force-displacement curve, and $P=\Psi(\delta)$ is the auxiliary one.

Expression $P(\delta)$ cannot be written explicitly. The only known expression for $P$ (equation (15)) contains $\bar{a}$. Therefore, $\bar{a}$ becomes new independent variable in (16) by means of change of variables $\delta=\delta_{c} f_{1}\left(\bar{a}, \delta_{c}, P_{c}\right)$ which is the first expression from (15).

As the result of this, the objective functional of the problem becomes

$$
\hat{\boldsymbol{\Phi}}\left(P_{c}, \delta_{c}\right)=\delta_{c} \int_{\bar{a}_{\min }}^{\bar{a}_{\max }}\left[P_{c} f_{2}\left(\bar{a}, \delta_{c}, P_{c}\right)-\Psi\left(\delta_{c} f_{1}\left(\bar{a}, \delta_{c}, P_{c}\right)\right)\right]^{2} \frac{\partial f_{1}\left(\bar{a}, \delta_{c}, P_{c}\right)}{\partial \bar{a}} d \bar{a}
$$

Since $\bar{a}$ has become a new independent variable, the problem of finding $\delta_{c}, P_{c}$ now has dimension two:

$$
\left\{P_{c}^{*}, \delta_{c}^{*}\right\}=\arg \min \hat{\boldsymbol{\Phi}}\left(P_{c}, \delta_{c}\right) .
$$

The expressions (17)-(18) establish the optimization problem of the extended BG method with pre-fitting.

In the Appendix A the reader can find a number of mathematical models known in the literature, which describe indentation of coated medium, and therefore have important practical applications. One of those models, developed by I. Argatov et al. [27] is used in the present work to numerically simulate the performance of the extended BG method. the model was chosen for benchmarking purposes, as a complex and computationally intensive one, yet simple enough to quickly implement it in a code for the Matlab software (MathWorks, Inc., Natick, MA, USA). 


\section{The extended BG method: numerical simulations and processing ex- perimental data}

Here the results of a number of numerical simulations and the results of processing a real experimental data are presented. The purpose of the calculations shown here is to examine accuracy, robustness and convergence speed of the proposed approaches.

In the recent years a number of papers regarding depth-sensing indentation of elastic medium covered with layers of another material has been published (see the previous subsection). In view of practical importance of this kind of the problems, the proposed extensions to the BG method were first tested in application to the model of contact between a spherical indenter and an elastic layer bonded to an elastic half-space [27]. In the case of spherical indenter the smallness of the ratio of the contact radius to the coating thickness necessary leads to the requirement of small indentation depth. Hence, in Appendix B we demonstrate that even at small indentation depths the substrate effect, that emerges due to the difference in the properties of the layer and the half-space, is not negligible.

In numerical simulations, the theoretical force-displacement curve obtained using expressions (33) and the characteristic parameters (37) was contaminated by noise and the data obtained were used to simulate the noisy experimental readings.

Clearly, the choice of particular model has influence on the overall result, when a real experiment is carried out. However, building sufficiently accurate model is not the part of the BG method, and is beyond the scope of the manuscript. To avoid the influence of the possible inaccuracy of the model, we used the model itself to generate the test data for simulations, thus testing only the ability of the BG method to re-create the initially set values of simulation parameters, while feeding a noisy data to it. In this case the simulation workflow was as follows:

1. Set the values of $E^{*}$ and $w$.

2. Calculate the corresponding values of $P_{c}$ and $\delta_{c}$, and build the theoretical load-displacement curve.

3. Introduce noise into the theoretical data.

4. Apply the BG method to contaminated data, identify $P_{c}$ and $\delta_{c}$.

5. Re-calculate $E^{*}$ and $w$ from the identified $P_{c}$ and $\delta_{c}$, compare to the initially set values.

When numerical simulations were carried out, calculation of the Tabor-Muller parameter was done immediately after setting the simulation parameters. Hence, we had a priori knowledge that the JKR theory was valid for these simulations, and no further checks were performed. However, it was not the case when the real experimental data was processed (Section 4.3). In that study the choice of the JKR theory to describe the experiment was validated a posteriori. See Section 4.3 for the details.

\subsection{Convergence speed and accuracy of the proposed approaches}

In order to investigate accuracy and the convergence speed of the both extended formulations of the BG method in the form (11) and (17), a data set of 100 data 
points was generated using the above mentioned mathematical model for adhesive contact between a rigid spherical indenter and a coated elastic medium (Appendix A). The $P$ and $\delta$ values were contaminated by means of adding zero-mean normally distributed noise with standard deviations equal to 0.03 of maximum values of $P$ and $\delta$ in the data set. The model parameters were taken as follows: $R=3 \mathrm{~mm}$, $h=1 \mathrm{~mm}, E_{2}=100 \mathrm{MPa}, \nu_{1}=0.3, \nu_{2}=0.25$.

Both the re-formulated BG approaches (11) and (17) were applied and the values of $E_{1}^{*}$ and $w$ were identified twice: (i) all the data points were used (including those corresponding to the tensile part of the $P-\delta$ curve), (ii) only the "compressive" part of the noisy data $(P>0)$ was used. The expected values were: $w=106.1$ $\mathrm{mJ} / \mathrm{m}^{2}, E_{1}^{*}=7.906 \mathrm{MPa}$.

The results of application of the direct fitting approach (11) are illustrated in Figure 4, where dots represent noise-contaminated data points, solid line is the reference force-displacement curve corresponding to the initially set values $w=106.1$ $\mathrm{mJ} / \mathrm{m}^{2}, E_{1}^{*}=7.906 \mathrm{MPa}$. The dashed line is the re-constructed force-displacement curve corresponding to the values of $E_{1}^{*}$ and $w$ identified by means of the BG method. Figure 4a shows application of the BG method (11) to the full set of data points (the identified material properties are $w=109.2 \mathrm{~mJ} / \mathrm{m}^{2}, E_{1}^{*}=7.939 \mathrm{MPa}$ ). Figure $4 \mathrm{~b}$ shows application of the BG method (11) to the compressive part of the data only (the identified material properties are $w=125.7 \mathrm{~mJ} / \mathrm{m}^{2}, E_{1}^{*}=8.347 \mathrm{MPa}$ ).

The similar results of application of the BG method with pre-fitting (17) are illustrated in the Figure 5. Pre-fitting was done using a 5-segment polygonal chain (that is, a linear piece-wise polynomial) in the framework of the orthogonal distance fitting approach. Figure 5a illustrates application of the BG method (17) to the full set of data points (the identified material properties are $w=105.1 \mathrm{~mJ} / \mathrm{m}^{2}$, $E_{1}^{*}=7.832 \mathrm{MPa}$ ). Figure 5b illustrates application of the BG method (17) to the compressive part of the data only (the identified material properties are $w=88.3$ $\left.\mathrm{mJ} / \mathrm{m}^{2}, E_{1}^{*}=7.758 \mathrm{MPa}\right)$.

The choice of the pre-fitting line as simple as a polygonal chain allowed us to explicitly program a Matlab function that evaluated sum of squared orthogonal distances from individual data points to the pre-fitting line. Minimization of that function led to optimal orthogonal fitting the polygonal chain to the data points (Figure 6). In particular, the polygonal chain was supposed to have $N_{S}$ segments and $N_{S}+1$ vertices. The first vertex had abscissa $\delta_{\min }=\min \left(\delta_{1}, \ldots, \delta_{N}\right)$, the last one had abscissa $\delta_{\max }=\max \left(\delta_{1}, \ldots, \delta_{N}\right)$ (see Figure 3$)$. The abscissas of the vertices were uniformly spaced: the $j$-th vertex abscissa was $\delta_{V j}=\delta_{\min }+\left(\delta_{\max }-\delta_{\min }\right)(j-$ $1) / N_{S}$. The ordinates of the vertices $P_{V j}$ were subject to ODF fitting in the space of dimensionless quantities as follows.

Since the indenter displacement and the indentation force are quantities of different physical nature and orders of magnitude, one needs to use their dimensionless equivalents when distance-based fitting approaches are used. Hence, to do pre-fitting in the framework of the ODF approach, the following normalized quantities were used $(i, n=1, \ldots, N)$ :

$$
\begin{aligned}
& \overline{\delta_{n}}=\frac{\delta_{n}-\left\langle\delta_{i}\right\rangle}{\max \left(\delta_{i}\right)-\min \left(\delta_{i}\right)}, \\
& \overline{P_{n}}=\frac{P_{n}-\left\langle P_{i}\right\rangle}{\max \left(P_{i}\right)-\min \left(P_{i}\right)} .
\end{aligned}
$$


Here, $N$ is the overall number of measurements in the data set, and $\langle\cdot\rangle$ is the averaging operator:

$$
\left\langle x_{i}\right\rangle=\frac{1}{N} \sum_{i=1}^{N} x_{i} .
$$

The coordinates of vertices of the pre-fitting polygonal chain were computed in the space of the dimensionless quantities $\overline{\delta_{n}}$ and $\overline{P_{n}}$. Afterwards, they were recalculated back to the space of dimensional quantities by inverting the formulae (19) (Figure $6)$.

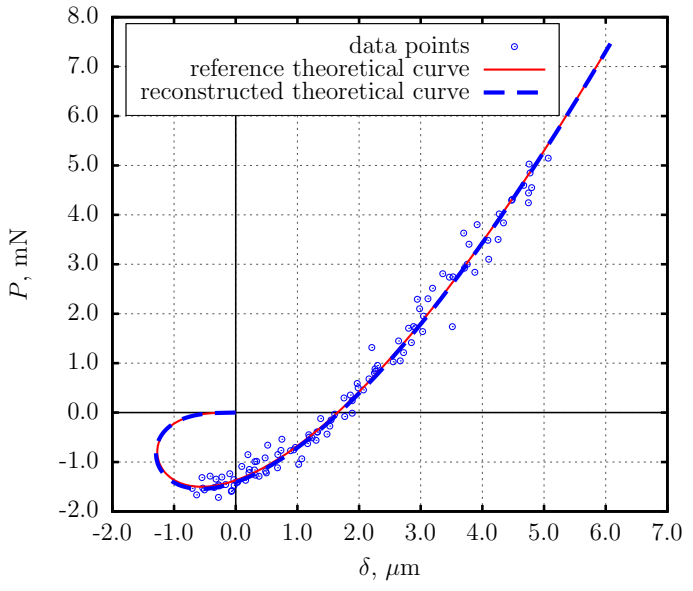

(a)

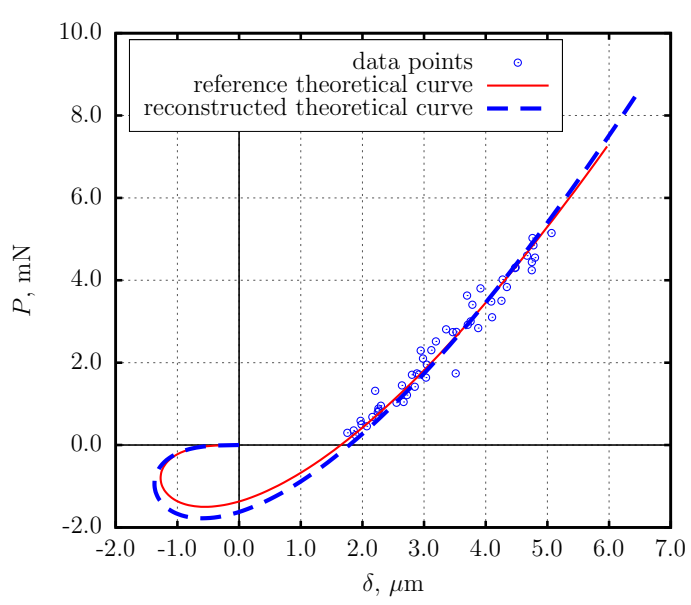

(b)

Figure 4: Numerical simulation. Reconstruction of the load-displacement curve from noisy data using the variant of the BG method (11). (a) Full unloading branch used. (b) Only compressive stage used.

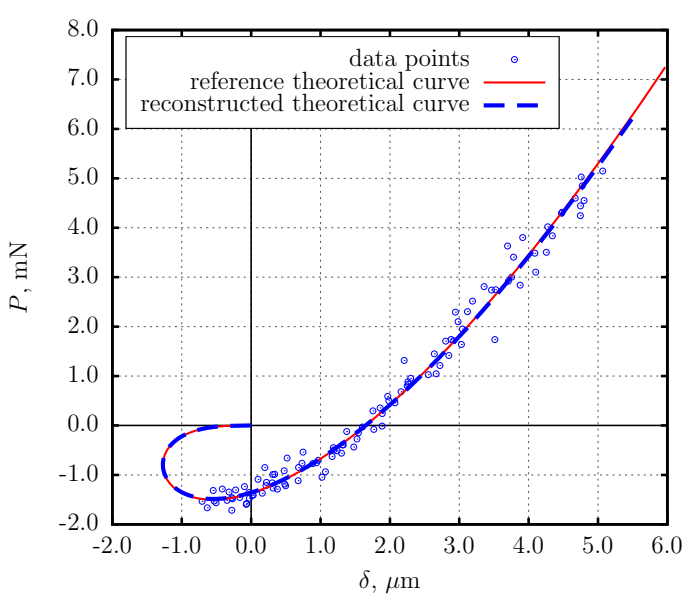

(a)

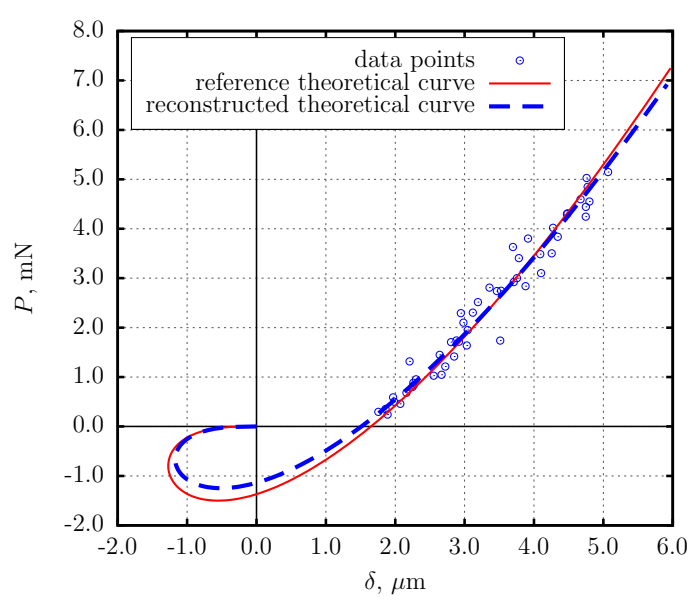

(b)

Figure 5: Numerical simulation. Reconstruction of the load-displacement curve from noisy data using the variant of the BG method (17). (a) Full unloading branch used. (b) Only compressive stage used.

The above numerical simulations allow us to conclude that both approaches (11) and (17) are very accurate when high enough number of data points is provided, and 


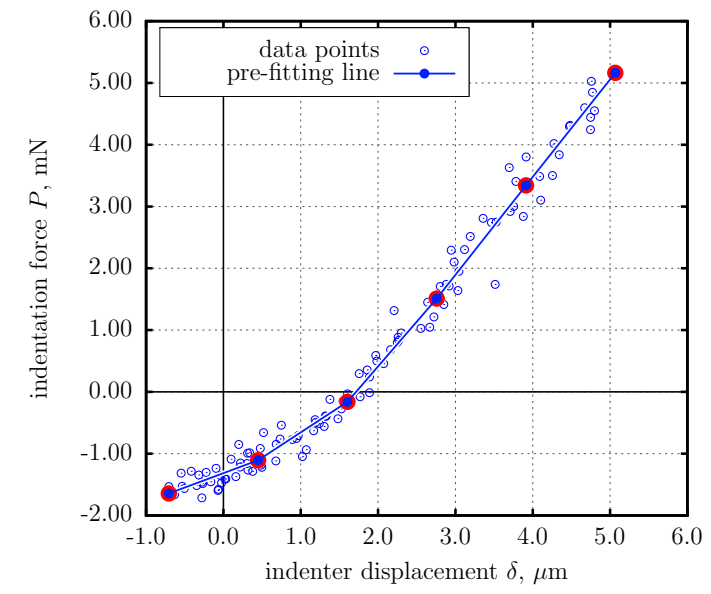

(a)

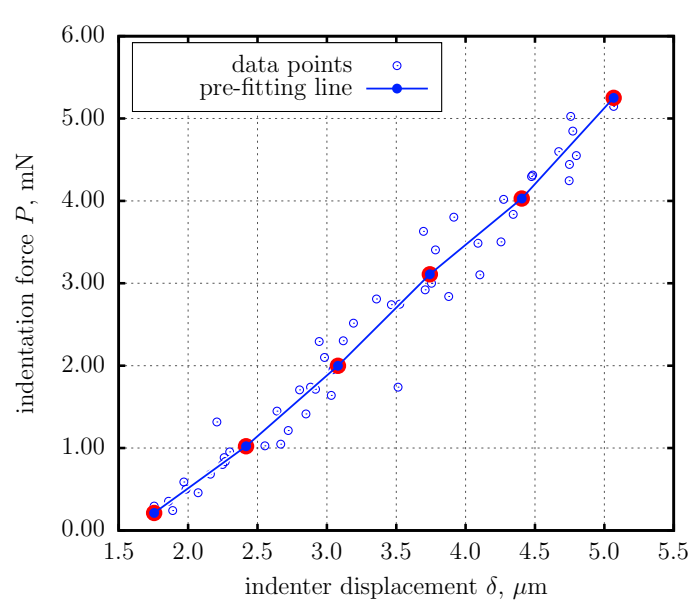

(b)

Figure 6: Numerical simulation. Preliminary fitting data points with a 5 segment polygonal chain. (a) Full unloading data used. (b) Compressive stage only used.

they cover sufficiently wide ranges of values of $P$ and $\delta$. A somewhat lower accuracy in the case of compressive data usage can be explained by two factors: (i) smaller range of $P$ and $\delta$ values, which makes the slope of the fitting curve more sensitive to the introduced noise, (ii) relatively small number of data points used in the simulation and their relatively high dispersion in comparison to the dimensions of data point clouds. The number of points has certain influence too because at relatively low number of points the data point cloud becomes less statistically representative, and, hence, fluctuations in individual points have greater influence on the result.

The more important conclusion is that introduction of an additional stage (prefitting) in data processing does not influence the accuracy level of the method much. However the analysis of convergence speed shows that the BG method with prefitting is tens to hundreds times faster than the direct fitting approach (11).

Consider Figure 7 in which convergence of the values of $P_{c}$ and $\delta_{c}$ is illustrated with respect to the number of iterations in the direct fitting approach (as described in the subsection 3.3). Figure 7a corresponds to the case when the full data set was used, while Figure $7 \mathrm{~b}$ corresponds to the case when only compressive data was used.

Given that the interleaving minimization algorithm explained in subsection 3.3 is similar to the coordinate descent method, such slow convergence is rather an expected and inevitable behaviour. At the same time the use of the BG method with pre-fitting required less than 20 iterations in each case.

Thus, the extended BG method with pre-fitting can be considered a robust, accurate and fast approach that can be recommended for processing large amounts of experimental data. This variant of the BG method is used in the rest of the present paper. In the next subsection the problem of unknown coordinate origin in experimental data is discussed. The performance of the BG method with pre-fitting is examined in the case when initial noisy data has an unknown coordinate origin. 


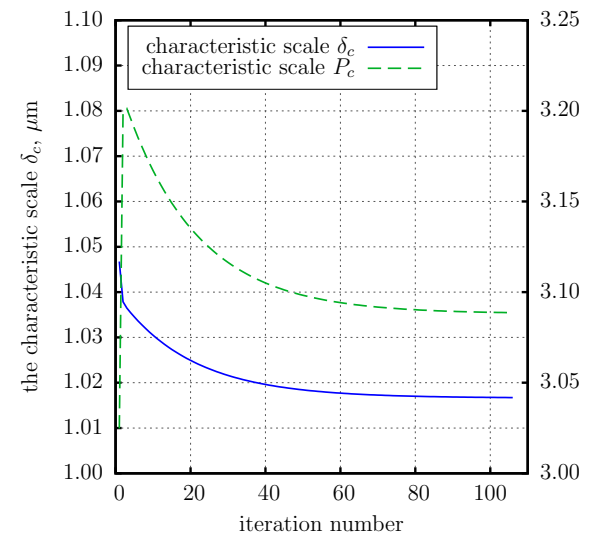

(a)

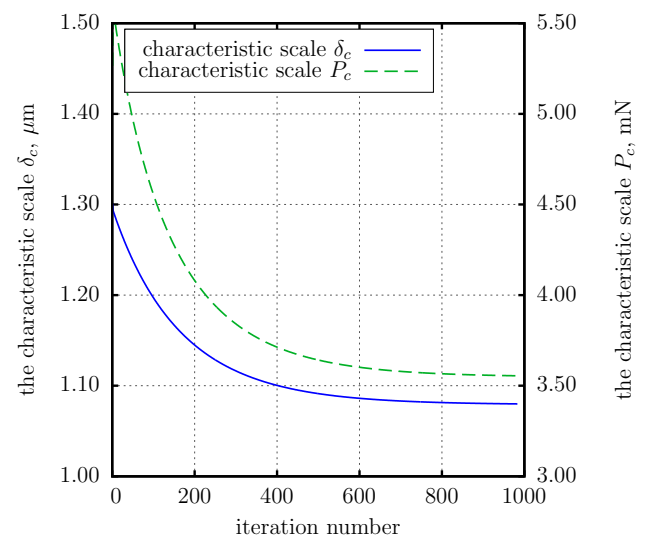

(b)

Figure 7: Numerical simulation. Convergence speed of the direct fitting approach (11). (a) Full unloading branch used. (b) Compressive stage only used.

\subsection{Processing experimental data: the problem of unknown coordinate origin}

Usually, the indentation equipment records position of the indentation tool using its own hardware-related reference point in which the displacement is considered to be zero. On the other hand, from a mathematical point of view the reference points (coordinate origins) of indentation curves in adhesive (the JKR theory) and nonadhesive (the Hertz theory) cases coincide and correspond to such a hypothetical position of the indenter in which the indenter and the sample touch at a single point and no intermolecular forces are taken into account (Figure 8a). Such a difference between the hardware coordinate origin and the theoretical one requires some shift value $\delta_{s}$ to be subtracted from the raw experimental data (or added to the abscissas of the theoretical curve) in order to obtain the right position of the indentation tool and correct data fitting (Figure 9).

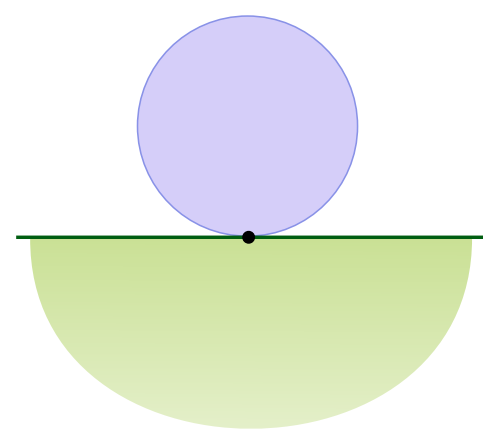

(a)

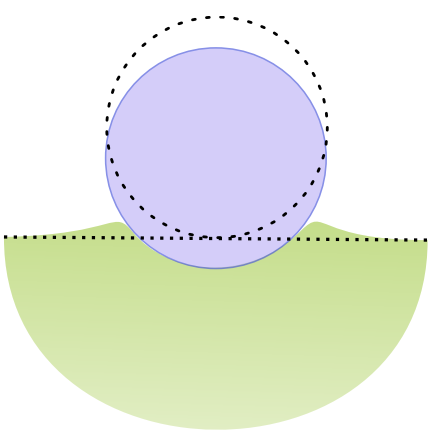

(b)

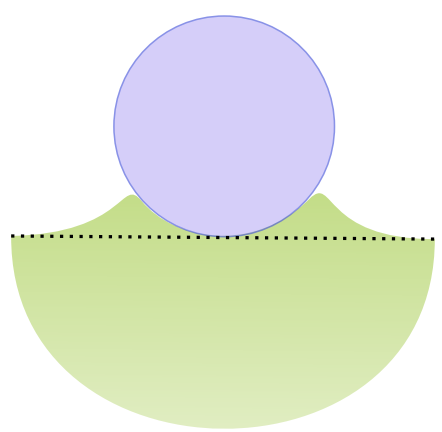

(c)

Figure 8: Relative positioning of the indenter and the specimen. (a) Position corresponding to theoretical coordinate origin $P=\delta=0$. (b) The real position when $P=0$. (c) The real position when $\delta=0$.

However, identification of such a shift value $\delta_{s}$ may be problematic. Indeed, the location of the point of initial contact between the indenter and the specimen is $a$ priori unknown as well as the actual distance between the specimen surface and the indenter surface at any given moment of time. Moreover, in reality the configuration 
depicted in (Figure 8a) cannot be achieved for micro-/nanoindentation due to the action of adhesive forces. If one measures zero indentation force, then the indenter already has some unknown displacement (Figure 8b). On the other hand, when indenter is in the right position, it is subject to some nonzero adhesive forces (Figure $8 \mathrm{c}$ ). Measurement noise and specimen surface imperfections also mask the moment of initial contact and make estimation of $\delta_{s}$ even harder.

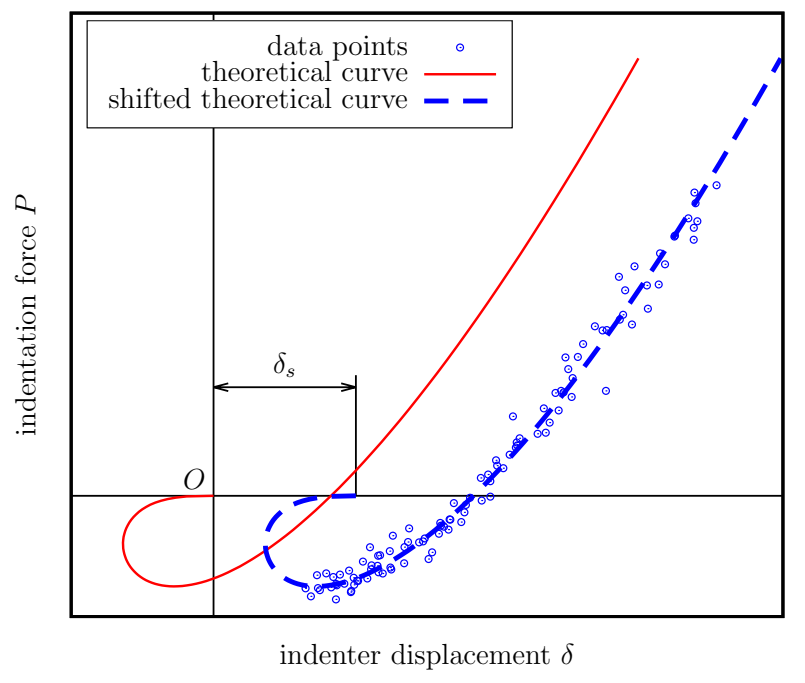

Figure 9: Illustration of the coordinate origin shift concept.

Hence, $\delta_{s}$ is an additional unknown variable in the data fitting process. The simplest way to identify this unknown quantity is as follows. For each experimental data set it is suggested to solve a number of trial problems of type (17)-(18). In the $k$-th trial problem the following data set is used for fitting:

$$
\delta_{i}^{(k)}=\delta_{M i}-\delta_{s}^{(k)}, \quad P_{i}^{(k)}=P_{M i}, \quad(i=1 \ldots N)
$$

where $\delta_{M i}$ and $P_{M i}$ denote the $i$-th pair of measured values of displacement and force correspondingly, and $\delta_{i}^{(k)}$ and $P_{i}^{(k)}$ stand for the $i$-th pair of displacement and force values in the $k$-th trial problem. The correct value of $\delta_{s}$ is supposed to give absolute minimum of the cost functional (17) among all the trial problems.

To verify this approach a set of six data sets (Figure 10) was generated using the asymptotic mathematical model of contact between an elastic layer bonded to an elastic half-space and a rigid punch (Appendix A).

Each of the sets emulates experimental data obtained from a single experimental test. According to the algorithm described above, the theoretical load-displacement curve (the reference curve) was calculated, then normally-distributed noise was added to the reference curve so that six different data sets were obtained. The added noise was zero-mean with standard deviations equal to $7.5 \%$ of $|\min (\delta)|$ and $|\min (P)|$ for displacement and force values respectively. A random shift was introduced into the values of $\delta$ as well.

The values of $E_{1}^{*}$ and $w$ were identified using the approach (17)-(18) with prefitting performed independently for each set (5-segment polygonal chain was used for preliminary fitting). Expected values were $w=106.1 \mathrm{~mJ} / \mathrm{m}^{2}, E_{1}^{*}=7.906 \mathrm{MPa}$, the same as in the previous simulation. The results of identification were as follows. The values of $E^{*}$, in MPa: 7.905, 7.731, 7.896, 7.896, 7.821, 7.846; the values of $w$, 


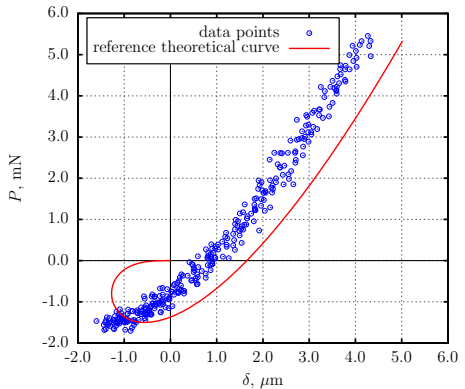

(a)

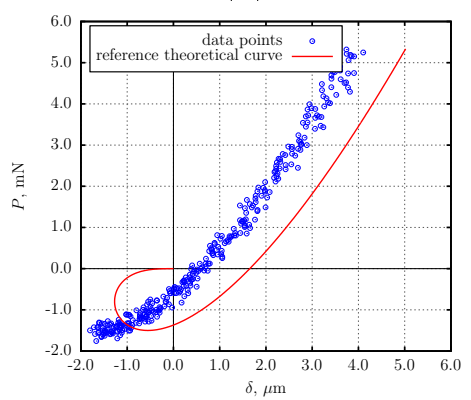

(d)

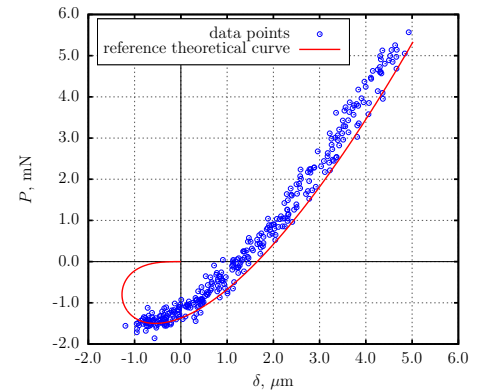

(b)

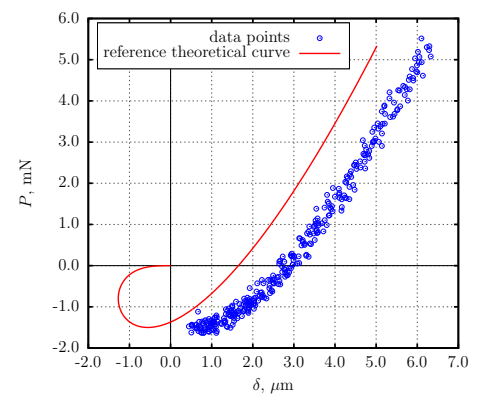

(e)

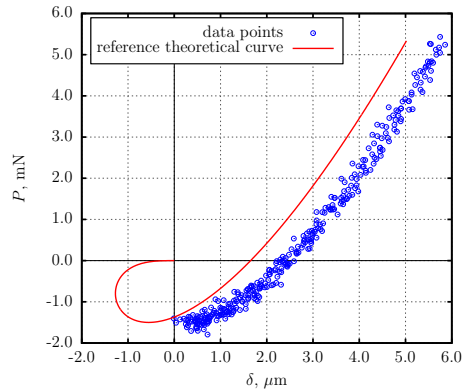

(c)

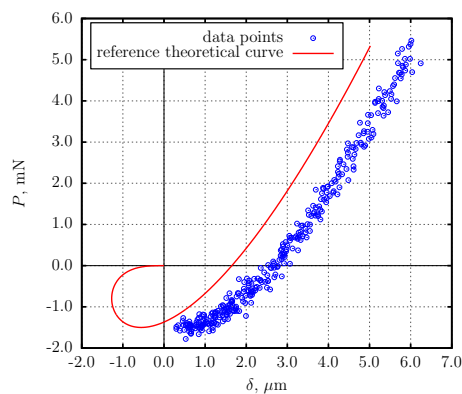

(f)

Figure 10: (a)-(f) Data sets (dots) with introduced random shifts in displacement readings $\delta$ which were used to simulate real experimental data. The solid line represents the reference theoretical curve used to obtain the data sets.

in $\mathrm{mJ} / \mathrm{m}^{2}$ : 110.2, 111.2, 109.8, 109.1, 107.7, 108.6. After averaging the following identified values of work of adhesion and effective elastic modulus were obtained: $w=109.4 \mathrm{~mJ} / \mathrm{m}^{2}, E_{1}^{*}=7.849 \mathrm{MPa}$. Relative errors therefore were $3.13 \%$ and $0.72 \%$ respectively. Standard deviations were $\sigma_{w}=1.233 \mathrm{~mJ} / \mathrm{m}^{2}, \sigma_{E^{*}}=0.06651$ MPa.

The shift value for each data set was identified during a two stage process. On the first stage the vicinity of the minimum of the objective functional with respect to shift in $\delta$ was found by means of the usage of linearly spaced set of the values of $\delta_{s}$. Then the value of $\delta_{s}$ was refined using the method of golden section. During each of these trial calculations the chosen value of $\delta_{s}$ was subtracted from the values of the data set (as suggested by (20)) and the functional (17) was minimized using built-in routines of Matlab software which resulted in the corresponding values of $P_{c}$ and $\delta_{c}$. The latter values were used for evaluation of $E_{1}^{*}$ and $w$ after the global minimum in the functional values with respect to $\delta_{s}$ had been reached.

\subsection{Identification of material properties from real experimental data}

To test the two-stage approach to identification of elastic and adhesive properties of materials with pre-fitting (see Eqs. (17)-(18)) and determination of unknown coordinate origin we used experimental data from the work by Borodich et al. [14].

In the paper [14], the BG method was used to identify properties of polyvinyl syloxane (PVS), a soft elastomer widely used as an impression material, in particular, in dentistry. Two series of six specimens were studied (with and without extra cleaning of the surface) using a custom-built force measurement device Basalt-1 (TETRA GmbH, Ilmenau, Germany). Each of the specimens was made of a thick 
PVS layer effectively considered as a half-space. Schematic of the experimental setup is depicted in Figure 11a. Indentation was carried out by means of sapphire balls of two different radii ( 1 and $3 \mathrm{~mm}$ balls used for each specimen) installed on the end of a cantilever beam of known stiffness. The position of the opposite end of the cantilever beam was controlled electronically by means of a piezo-drive. Positions of the both ends of the cantilever were measured by means of optical sensors. These readings were then converted into the values of applied indentation force and the displacement of the indenter ball (Figure 11b). The values of the reduced contact modulus $E^{*}$ and the work of adhesion $w$ identified in [14] are used here (see Figure 14, Table 1) as the reference data to confirm accuracy of the extended BG method.

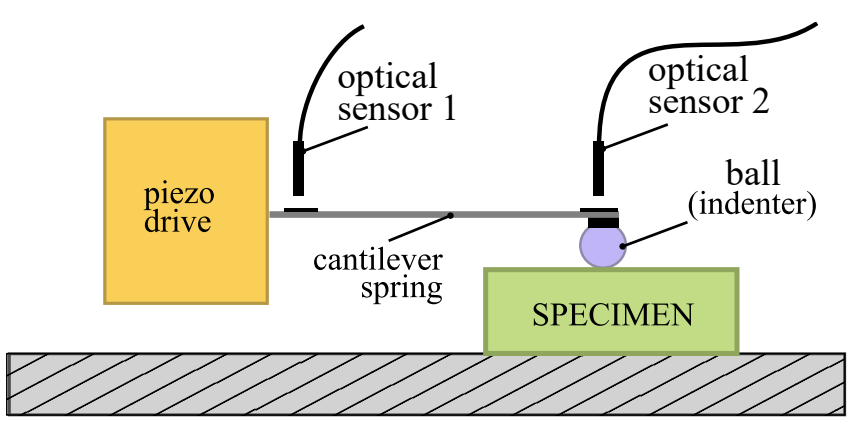

(a)

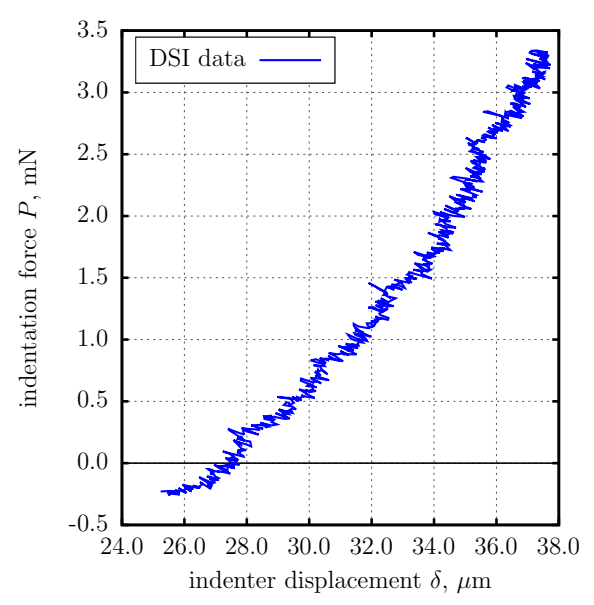

(b)

Figure 11: (a) Schematic of the DSI setup. (b) An example of experimental forcedisplacement curve.

Two series of six specimens each tested with 1 and $3 \mathrm{~mm}$ balls produced 24 data sets in total. In the present work, each individual data set was processed using the extended BG approach (17)-(18) as follows.

Due to small indentation depth, the classical JKR theory of adhesive contact interaction between a rigid sphere and an elastic half-space was used. The particular representation of the theoretical force-displacement curve was chosen in the present work in the form (4). The stable branch of (4) was re-written in parametric form as

$$
\left\{\begin{array}{l}
\delta=\delta_{c}(3 \sqrt{1+\bar{a}}-1)\left(\frac{1+\sqrt{1+\bar{a}}}{9}\right)^{\frac{1}{3}}, \\
P=P_{c} \bar{a}
\end{array}\right.
$$

These expressions became particular form of the formulae (15) used to process experimental data. The characteristic parameters $P_{c}$ and $\delta_{c}$ were defined using formulae (5).

Preliminary fitting of experimental data was done by means of polygonal chain which was used as the pre-fitting function $P=\Psi(\delta)$ in (17). Since the number of segments in the pre-fitting polygonal chain has some influence on the identified values of $E^{*}$ and $w$, the number of segments was varied from 3 to 10 . Every time the material properties were identified separately for each of the 24 data sets. As the result of statistical post-processing, the averaged values $\left\langle E_{i}^{*}\right\rangle$ and $\left\langle w_{i}\right\rangle$ as well as the standard deviations $\sigma_{E^{*}}$ and $\sigma_{w}$ were computed. 
The dependency of the averaged values of the reduced elastic contact modulus and the work of adhesion on the number of segments is shown in Figure 12a. The averaged values of $E^{*}$ vary from 2.248 to $2.274 \mathrm{MPa}$, the averaged values of $w$ vary from 55.18 to $59.56 \mathrm{~mJ} / \mathrm{m}^{2}$. The dependency of the values of standard deviations $\sigma_{E^{*}}$ and $\sigma_{w}$ on the number of segments is shown in Figure $12 \mathrm{~b}$.

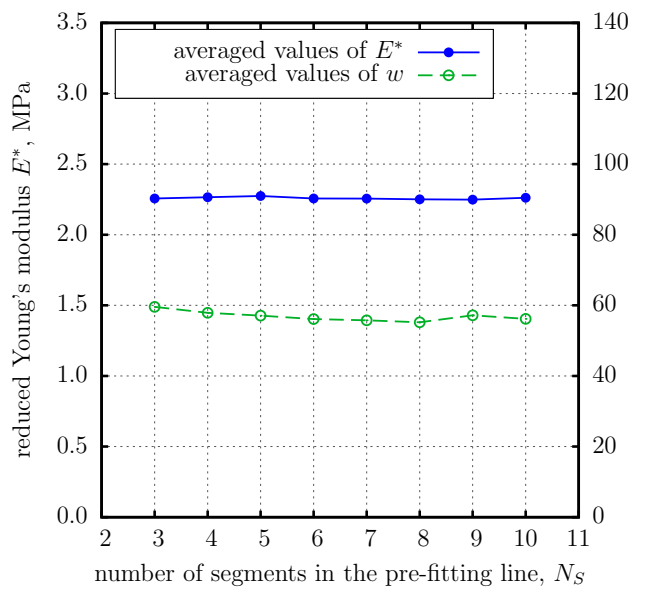

(a)

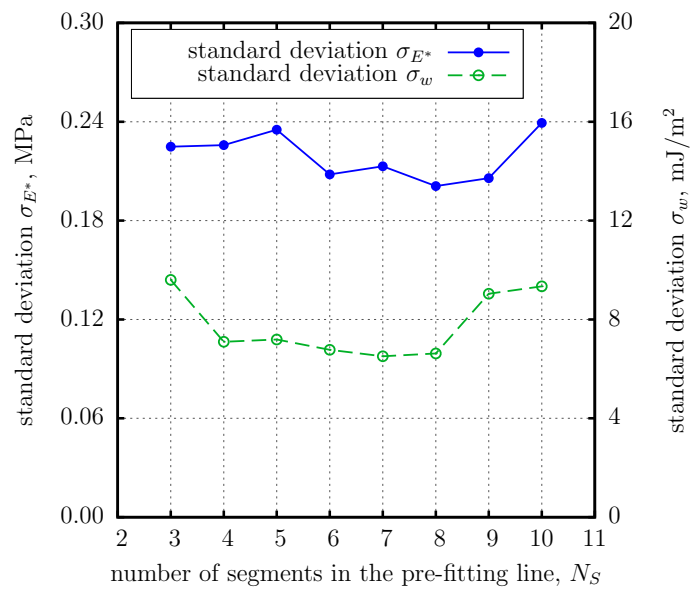

(b)

Figure 12: The results of processing of the raw experimental force-displacement DSI data after the paper by Borodich et al. [14]. (a) Averaged values of reduced elastic contact modulus and the work of adhesion versus the number of segments in the pre-fitting curve. (b) Standard deviations of the identified PVS properties values versus the number of segments in the pre-fitting curve.

To identify the optimal number of segments in the pre-fitting line, the following dimensionless measures can be used. The goodness of identification of individual material properties can be described by the corresponding relative standard deviations (coefficients of variation) defined as

$$
c_{v E^{*}}=\frac{\sigma_{E^{*}}}{\left\langle E_{i}^{*}\right\rangle}, \quad c_{v w}=\frac{\sigma_{w}}{\left\langle w_{i}\right\rangle}
$$

where $c_{v E^{*}}$ and $c_{v w}$ are relative standard deviations for $E^{*}$ and $w$ correspondingly.

Optimal settings should produce minimal scatter of the identified values of material properties. However, in the present study the computed values of $c_{v E^{*}}$ and $c_{v w}$ reached minimum at different number of segments in the pre-fitting line (Figure 13). Hence, generalized variance was used as a single scalar measure of overall multidimensional scatter of points corresponding to identified values of $E^{*}$ and $w$ on the $\left(w, E^{*}\right)$ plane. Generalized variance $|\Sigma|$ is defined as the determinant of the covariance matrix $\Sigma\left(E_{i}^{*}, w_{i}\right)$ of random quantities $E^{*}$ and $w[59,60]$ :

$$
|\Sigma|=\operatorname{det}\left(\Sigma\left(E_{i}^{*}, w_{i}\right)\right)
$$

In the present study generalized variance was computed using normalized data thus producing a dimensionless metric of goodness of fit:

$$
|\Sigma|^{*}=\operatorname{det}\left(\Sigma\left(\frac{E_{i}^{*}}{\left\langle E_{i}^{*}\right\rangle}, \frac{w_{i}}{\left\langle w_{i}\right\rangle}\right)\right) \text {. }
$$




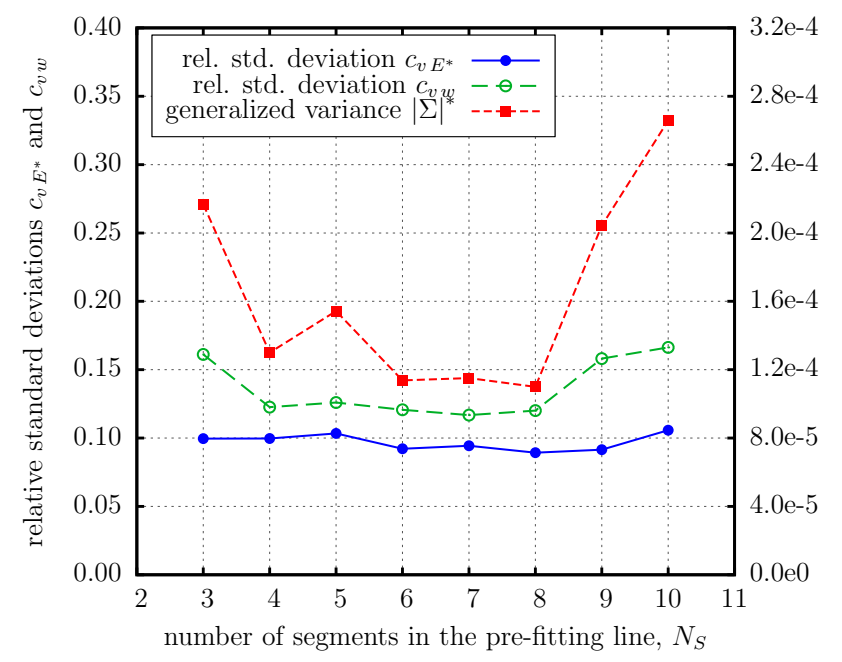

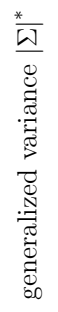

Figure 13: Dimensionless metrics describing goodness of fit versus the number of segments in the pre-fitting curve: relative standard deviations $c_{v E^{*}}, c_{v w}$ and generalized variance $|\Sigma|^{*}$

The dependency of $|\Sigma|^{*}$ on the number of segments $N_{S}$ in the pre-fitting line is shown in Figure 13. It reached minimum at $N_{S}=8$. Thus, $N_{S}=8$ was considered as the optimal number of segments producing best fit for the data used.

In Figure 14a the results of identification are shown for pre-fitting with 8-segment line. These results were considered optimal and compared with the values of $E^{*}$ and $w$ obtained in [14] by means of the original BG method (Figure 14b). In Table 1 the statistical measures corresponding to the two cases are presented: the averaged value of the reduced contact modulus $\left\langle E_{i}^{*}\right\rangle$, the averaged value of the work of adhesion $\left\langle w_{i}\right\rangle$, and the corresponding standard deviations $\sigma_{E^{*}}$ and $\sigma_{w}$. The relative differences in Table 1 were computed as

$$
\Delta_{r e l}=\frac{\left|Q_{B G}^{e x t}-Q_{B G}^{\text {orig }}\right|}{Q_{B G}^{\text {orig }}}
$$

where $Q$ denotes one of the physical quantities listed above, superscripts "ext" and "orig" denote the extended implementation of the BG method (17)-(18) and the original one [14] correspondingly. The complete result set for the number of segments varied from 3 to 10 is shown in Appendix C.

Comparison of the results produced by the original and the extended BG methods leads to an important conclusion that at optimal settings the extended BG method produces very similar averaged values of the material properties, and yet it has better performance in terms of lesser scatter of the identified values (i.e. lower standard deviation values).

Indeed, the considered DSI tests fall within the range of applicability of the JKR theory. Tabor [61] and Muller et al. [62] (see also [39]) introduced a dimensionless parameter:

$$
\mu=\left(\frac{R w^{2}}{E^{* 2} z_{0}^{3}}\right)^{1 / 3}
$$

where $R$ is the effective curvature radius of contacting bodies (if a spherical indenter is in contact with a plane, $R$ is equal to the radius of the sphere); $z_{0}$ is the equilibrium 
distance between atoms of the contacting bodies, usually assumed to be $0.3 \ldots 0.5 \mathrm{~nm}$. This parameter is suitable for clear distinction of applicability boundary between the JKR and DMT theories of adhesive contact.

Assuming $z_{0}=0.4 \mathrm{~nm}$ and using the identified total maximum and minimum values of the reduced elastic modulus (2.69 and $1.85 \mathrm{MPa}$ correspondingly), the work of adhesion ( 63.3 and $39.8 \mathrm{~mJ} / \mathrm{m}^{2}$ correspondingly), and the indenter radii, one can estimate the range of the Tabor-Muller parameter as $1506 \leq \mu \leq 3800$ which is much greater than the threshold value $\mu=1$. This justifies the use of the JKR theory.

Remark. In the recent work by Perepelkin et al. [20] the proposed extended variant of the $B G$ method with pre-fitting has been experimentally validated. Two types of specimens were made of the same charge of PVS, and two different experiments were carried out: (i) depth sensing indentation using the experimental set-up shown in Figure 11a (including post-processing of the experimental data by means of the extended BG method), (ii) tensile testing of dumbbell-shaped specimens as a reference experiment. Values of the reduced elastic modulus $E^{*}$ estimated in both experiments were in good agreement. Note that these values differ from presented in Table 1 because mechanical properties of PVS may vary between different charges of the material.

Table 1: Comparison of the experimental results from Borodich et al. and the present work

\begin{tabular}{cccc}
\hline Quantity & Borodich et al. (2013) & $\begin{array}{c}\text { The present work } \\
\text { (at optimal fitting } \\
\text { settings) }\end{array}$ & $\begin{array}{c}\text { Relative } \\
\text { difference, } \%\end{array}$ \\
\hline$\left\langle E_{i}^{*}\right\rangle, \mathrm{MPa}$ & 2.39 & 2.25 & 5.86 \\
\hline$\left\langle w_{i}\right\rangle, \mathrm{mJ} / \mathrm{m}^{2}$ & 57.6 & 55.2 & 4.16 \\
\hline$\sigma_{E^{*}}, \mathrm{MPa}$ & 0.204 & 0.201 & 1.47 \\
\hline$\sigma_{w}, \mathrm{~mJ} / \mathrm{m}^{2}$ & 8.51 & 6.62 & 22.2 \\
\hline
\end{tabular}

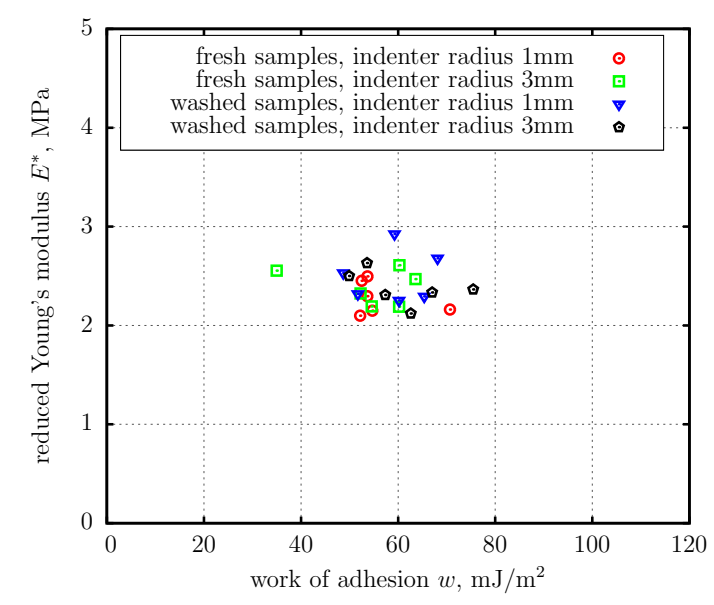

(a)

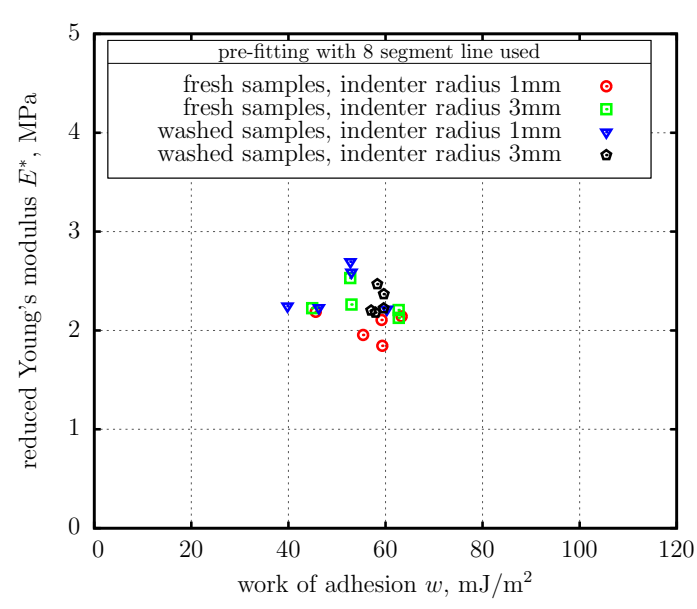

(b)

Figure 14: Comparison of the experimental results after Borodich et al. [14] and the present work. (a) The values of $E^{*}$ and $w$ identified in [14]. (b) The set of identified values of material properties extracted using pre-fitting with polygonal chain. Number of segments in chain: 8 (optimal value). 


\section{Conclusions}

To identify correctly material properties from micro- or nano-indentation, the corresponding mathematical models should take into account adhesion between the indenter and the specimen. Here we have extended the BG method to a general class of mathematical models of adhesive contact when the external load and the displacement of the indenter are defined as parametric functions of the contact radius. In particular, one can use any well-established model (e.g. [49]) of adhesive contact for an elastic layer and apply the extended BG method to experimental data obtained by DSI of layered/coated medium in order to extract physical properties such as the work of adhesion, contact elastic modulus, and so on.

To fit experimental data with a parametric force-displacement curve we have introduced a new objective functional of the BG method based on the concept of orthogonal distance curve fitting (ODF). However, fitting a parametric $P-\delta$ curve to a set of points normally requires to determine the unknown values of the parameter corresponding to individual data points which increases the number of unknowns drastically and slows down computations. To address this issue of the above direct fitting approach the following additional extension of the BG method has been suggested. It has been proposed to carry out fitting of the theoretical force-displacement curve to experimental data points by means of a two-stage process. On the preliminary first stage (the pre-fitting stage) data points are fitted with an auxiliary curve. The auxiliary curve is supposed to have simplest possible mathematical form which allows to implement advanced data fitting/filtering techniques like ODF quickly and effectively. The pre-fitting curve has been taken as simple as a polygonal chain which is fitted to experimental data using the ODF technique. On the second stage the theoretical force-displacement curve is fitted to the auxiliary one by means of minimization of the square of the norm of difference of the two functions. The objective functional has been formulated in a way that does not require computation of unknown values of the parameter. Numerical simulations showed that the prefitting stage does not have significant influence on the accuracy of identification of the values of the reduced contact modulus and the work of adhesion.

The extended BG method with pre-fitting was tested using real experimental data from DSI experiments [14] in which the original BG method was used. The values of the reduced contact modulus $E^{*}$ and the work of adhesion $w$ of polyvinyl siloxane samples were identified using the extended BG method in the framework of the classic JKR theory and compared to the previously published ones. Comparison showed that the averaged values of material properties obtained by means of the extended BG method at the optimal pre-fitting settings differ insignificantly from the values obtained by means of the original $B G$ method. This means that the extended BG method is at least as good as the original BG method, while the individual values of material properties produced by the eBG method were less scattered and therefore, having lower standard deviation values in the resulting data set.

The parametric models of adhesive contact include such important cases as indentation of thin and ultra-thin layers and coatings, cell membranes, etc. The applications of the extended BG method to many of these models have been discussed. Numerical simulations were carried out using an asymptotic mathematical model for adhesive contact between a rigid axisymmetric indenter and a thick elastic layer bonded to an elastic half-space developed by Argatov et al. [27]. Numerical 
simulations confirmed the accuracy and robustness of the proposed extensions of the BG method against data contaminated by normally-distributed noise.

Thus, the BG and eBG methods may be considered as a framework for extracting mechanical and physical characteristics of contacting solids based on a combination of indentation techniques and numerical approaches to processing experimental data. From this point of view, the BG method is much more than just a fitting procedure.

\section{Funding}

This work was supported by the European Union's Horizon 2020 research and innovation programme under the Marie Skłodowska-Curie grant agreement No 663830

\section{Acknowledgements}

The collaboration between two of the authors (IA and FB) on the topic of the paper was initiated within the CARBTRIB International network. The authors are grateful to the Leverhulme Trust for support of the Network.

The authors express their gratitude to Dr. Andrey Pepelyshev (Cardiff University) for his valuable comments to the present work.

\section{Appendix A. The scope of the extended BG method in application to parametric $P-\delta$ curves. Coated medium}

In this Appendix we briefly list a number of models, which have important practical applications, all have similar structure, and thus can be used in the same manner with the extended BG method. Here we focus on the models that a mathematically formulated in the form of parametric functions.

The classic JKR theory considers contact between a sphere and an elastic halfspace. In the case of sphere the JKR theory leads to an explicit relation $P(\delta)$. However, if one considers more general shapes of the indenters [8] the $P-\delta$ curve may be presented only in the parametric form $(P(a), \delta(a))$ where $a$ is the contact radius. This type of representation is very important. In particular (but not limited to), such parametric dependencies may arise, for example, as the result of extension of a $P-\delta$ dependency from non-adhesive case to adhesive one in the framework of the JKR theory of adhesive contact. As an important case in which the proposed extensions of the BG method can be used one may consider indentation of a coated or layered medium.

It is important to emphasize that different sets of characteristic parameters are used by different authors to represent the force-displacement relations in dimensionless form (see e.g. discussion in $[8,63]$ ). These parameters $a_{c}, P_{c}, \delta_{c}$ are used to construct the dimensionless contact radius $\bar{a}=a / a_{c}$, indentation force $\bar{P}=P / P_{c}$, and indenter displacement $\bar{\delta}=\delta / \delta_{c}$.

Apart from the above mentioned formulae (5), one can find the following examples of characteristic parameters. In the works by Johnson [64], and Maugis [39] the following set of parameters is suggested:

$$
a_{c}=\left(\frac{3 \pi w R^{2}}{4 E *}\right)^{1 / 3}, \quad P_{c}=\pi w R, \quad \delta_{c}=\left(\frac{9 \pi^{2} w^{2} R}{16 E^{* 2}}\right) .
$$


The paper by Johnson and Sridhar [49] suggests slightly different set of characteristic parameters:

$$
a_{c}=\left(\frac{9 \pi w R^{2}}{4 E *}\right)^{1 / 3}, \quad P_{c}=\pi w R, \quad \delta_{c}=\left(\frac{9 \pi^{2} w^{2} R}{16 E^{* 2}}\right) .
$$

The above examples suggest that reader should be careful while considering various force-displacement dependencies, as the used characteristic parameters may differ from one dependency to another.

Indentation of a thin elastic layer bonded to a rigid base. It is known [65-68] that a thin elastic layer in a contact problem can be effectively reduced in leading-term approximation to the spring-bed foundation (the Winkler foundation) defined through its elastic constant $K$ which in isotropic limit can be expressed as $K=E(1-\nu) /[h(1+\nu)(1-2 \nu)]$ where $E$ is the elastic modulus and $\nu$ is the Poisson's ratio of the layer material, and $h$ is the layer thickness. Hence, the JKR adhesive solution for a contact problem for an arbitrary convex rigid axisymmetric indenter and a thin elastic layer bonded to the rigid substrate can be expressed as [43]:

$$
P=\pi K a^{2}\left(f(a)-\sqrt{\frac{2 w}{K}}\right)-2 \pi K \int_{0}^{a} f(r) r d r
$$

and

$$
\delta=f(a)-\sqrt{\frac{2 w}{K}} .
$$

The function $f(r)$ is an arbitrary function defining indenter shape in cylindrical coordinates. Clearly, the parameter $a$ (the contact radius) can be eliminated from (29)-(30) only in some particular cases.

Indentation of an elastic layer bonded to an elastic half-space. Semianalytical approach. Adhesive solutions of contact problems become even more complex if a coated elastic medium is considered. To address this issue, a semianalytical approach to solving the contact problem for a coated elastic medium and an axisymmetrical indenter was developed in [48-50]. For instance, Johnson and Sridhar [49] represent JKR force-displacement curves in dimensionless form as

$$
\bar{\delta}=3^{2 / 3} \bar{a}^{2}\left(B(x, k)-\frac{2 \sqrt{2}}{3} \bar{a}^{-3 / 2} D(x, k)\right)
$$

and

$$
\bar{P}=\bar{a}^{3} F(x, k)-\sqrt{2 \bar{a}^{3}} H(x, k)
$$

where $\bar{\delta}$ and $\bar{P}$ the dimensionless variables obtained using the characteristic parameters (28). They represent the indentation depth and force respectively. The dimensionless radius of the contact region $\bar{a}$ serves as the parameter along the $P-\delta$ curve. $B(x, k), D(x, k), F(x, k), H(x, k)$ are numerically computed correction functions that depend on the ratio of contact radius to coating thickness $x$ and the ratio of Young's moduli of substrate and coating $k$. Clearly, this kind of force-displacement curve cannot be exactly reduced to explicit/implicit function. 
Indentation of an elastic layer bonded to an elastic half-space. Asymptotic approach. Another example of parametrically formulated force-displacement curve is an analytical asymptotic model for adhesive contact between a spherical indenter and a thick elastic layer bonded to an elastic half-space. This asymptotic model was developed by Argatov and Sabina [44] for non-adhesive case and extended for JKR-type adhesive contact by Argatov et al. [27] (see also [69]). Let us consider this model in more detail as it is used in the present paper as the base for mathematical modelling.

Let a rigid spherical indenter of radius $R$ be in contact with an elastic medium covered with an elastic layer of thickness $h$ (Figure 15). Elastic modulus and Poisson's ratio for elastic medium are considered to be known $\left(E_{2}, \nu_{2}\right)$ as well as the Poisson's ratio of the layer $\nu_{1}$. Indenter is subject to force $P$, indentation depth $\delta$ is considered to me small in comparison with layer thickness $h$. Radius of contact area is denoted as $a$.

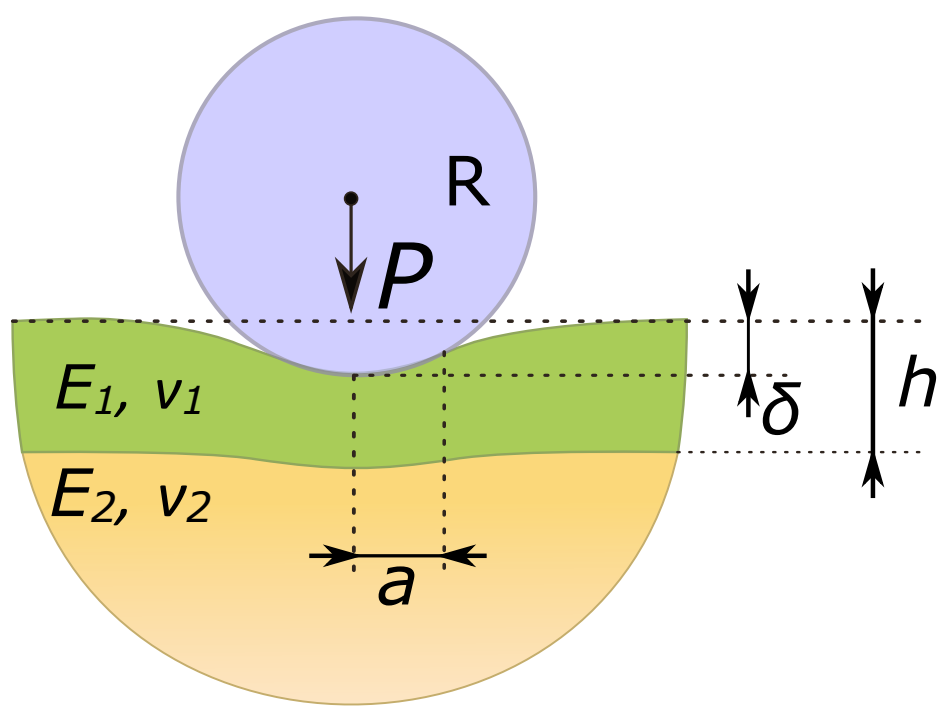

Figure 15: Indentation of an elastic layer bonded to an elastic half-space.

According to Argatov et al. [27], the load-displacement curve for spherical indenter can be expressed as an asymptotic expansion:

$$
\begin{aligned}
\bar{\delta} \simeq & 3 \bar{a}^{2}\left(1-\varepsilon \frac{4 a_{0}}{3 \pi}-\varepsilon^{3} \frac{16 a_{1}}{5 \pi}+\varepsilon^{4} \frac{32 a_{0} a_{1}}{9 \pi^{2}}\right) \\
& -2 \sqrt{2 \bar{a}}\left(1-\varepsilon \frac{2 a_{0}}{\pi}-\varepsilon^{3} \frac{16 a_{1}}{3 \pi}+\varepsilon^{4} \frac{16 a_{0} a_{1}}{3 \pi^{2}}\right) \\
\bar{P} \simeq & \left(1-3 a_{1} \alpha^{9 / 2} \bar{a}^{3}\right)\left(\bar{a}^{3}-\sqrt{2} \bar{a}^{3 / 2}\right)
\end{aligned}
$$

One can see that the relation between the dimensionless variables $\bar{P}$ and $\bar{\delta}$ is formulated again in the parametric form, where the dimensionless contact radius $\bar{a}$ is used as the parameter. Other coefficients can be expressed as follows:

$$
\alpha=\left(\frac{2 w R^{2}}{E_{1}^{*} h^{3}}\right)^{1 / 2}, \quad a_{m}=\frac{(-1)^{m}}{2^{2 m}(m !)^{2}} \int_{0}^{\infty} \mathcal{L}(u) u^{2 m} d u
$$


where

$$
\begin{aligned}
\mathcal{L}(u) & =\frac{2 K L e^{-4 u}-\left(L+K+4 u K+4 u^{2} K\right) e^{-2 u}}{1-\left(L+K+4 u^{2} K\right) e^{-2 u}+K L e^{-4 u}}, \\
K & =\frac{1-n}{1+n\left(3-4 \nu_{1}\right)}, \quad L=\frac{\left(3-4 \nu_{2}\right)-n\left(3-4 \nu_{1}\right)}{\left(3-4 \nu_{2}\right)+n}, \quad n=\frac{E_{2}\left(1+\nu_{1}\right)}{E_{1}\left(1+\nu_{2}\right)} .
\end{aligned}
$$

Small parameter $\varepsilon$ can be introduced as $\varepsilon=a / h$ or can be evaluated using the other model parameters as [27]:

$$
\varepsilon=\frac{\sqrt[3]{9 \pi}}{2} \alpha^{2 / 3} \bar{a}
$$

Unknown elastic modulus $E_{1}$ can be eliminated using expression $E_{1}^{*}=E_{1} /\left(1-\nu_{1}^{2}\right)$, therefore, the expressions above contain two unknown quantities: effective elastic modulus $E_{1}^{*}$ and the work of adhesion $w$.

As it was stated earlier, the BG method uses scaling relations to connect unknown material properties and characteristic scaling parameters $P_{c}$ and $\delta_{c}$. Mathematical model (33) was developed using the following scaling relations [27]:

$$
\bar{P}=\frac{P}{3 \pi w R}, \bar{\delta}=\delta\left(\frac{4 E_{1}^{*}}{\sqrt{3 R} \pi w}\right)^{2 / 3}, \bar{a}=a\left(\frac{4 E_{1}^{*}}{9 \pi w R^{2}}\right)^{1 / 3} .
$$

i.e. the characteristic parameters $P_{c}$ and $\delta_{c}$ can be expressed as:

$$
P_{c}=3 \pi w R, \delta_{c}=\left(\frac{\sqrt{3 R} \pi w}{4 E_{1}^{*}}\right)^{2 / 3} .
$$

Please note that $P_{c}$ and $\delta_{c}$ differ from the examples presented above in formulae (5), (27), and (28).

Therefore, once $P_{c}$ and $\delta_{c}$ are obtained via BG method, $E_{1}^{*}$ and $w$ can be evaluated:

$$
w=\frac{P_{c}}{3 \pi R}, E_{1}^{*}=\frac{P_{c}}{\sqrt{3 R} \delta_{c}^{3 / 2}} .
$$

This means that optimization problems (11) and (17) can be fully formulated in terms of $P_{c}$ and $\delta_{c}$.

Indentation of a functionally-graded half-space. Clearly, parametric representation of the solution of the adhesive contact problem is not limited to the scope of the JKR theory. E.g., Jin et al. [47] used the double-Hertz analytical model of adhesive contact by Greenwood and Johnson [70] to study the contact problem for a rigid spherical punch and a functionally graded elastic half-space with the elastic modulus $E$ varied through the depth $z$ as $E=E_{0}\left(z / c_{0}\right)^{k}$. According to [47], the solution of the contact problem was presented using the contact radius $a$ as the parameter as

$$
\begin{gathered}
P=\frac{2 \pi \alpha_{1}}{3+k} \frac{E^{*}}{c_{0}^{k} R}\left[a^{3+k}-\lambda\left(c^{3+k}-a^{3+k}\right)\right], \\
\delta=\frac{a^{2}-\lambda\left(c^{2}-a^{2}\right)}{R(1+k)},
\end{gathered}
$$




$$
w=\lambda(1+\lambda) \frac{\alpha_{1} E^{*}}{c_{0}^{k} R} \frac{(1+k) c^{3+k}-(3+k) a^{2} c^{1+k}+2 a^{3+k}}{(1+k)(3+k)} .
$$

The latter equation establishes the relation between the work of adhesion $w$, the outer radius of the cohesive zone $c$, and the rest of the parameters of the system.

Indentation of a coated elastic half-space. The Maugis-Dugdale approach. In their work, Sergici et al. [45] considered an adhesive contact problem for a spherical indenter and a coated half-space in the framework of the MaugisDugdale theory. In dimensionless form the expressions for the total applied force and the indendter displacement were defined as

$$
\bar{P}=-\pi \bar{a}^{2}\left(\int_{0}^{1} G(\bar{\varepsilon}) \bar{\varepsilon} d \bar{\varepsilon}+m^{2} \frac{\lambda}{2}\right)
$$

and

$\bar{\delta}=\frac{\bar{a}^{2}}{2}+\frac{8 \bar{a}}{3 \pi} \int_{0}^{1} \bar{\varepsilon} G(\bar{\varepsilon}) K(\bar{\varepsilon}) d \bar{\varepsilon}+\frac{4}{3} \bar{a}\left(\frac{\bar{a}}{H}\right) \int_{0}^{1} \bar{\varepsilon} G(\bar{\varepsilon}) k_{8}(\bar{\varepsilon}, 1) d \bar{\varepsilon}+\frac{8 \bar{a}}{3 \pi} \lambda m E(1 / m)+\frac{8 \bar{a}}{3} \lambda m k_{9}(1)$

where dimensionless quantities $\bar{a}, \bar{P}$, and $\bar{\delta}$ were obtained using the characteristic parameters (27), and $m$ is ratio of outer radius of the cohesive zone $c$ to the contact radius $a . G$ is the dimensionless distribution of the normal stresses in the contact area, and $k_{8}, k_{9}$ are auxiliary functions depending on the particular geometry of the problem and material properties. Note the dimensionless contact radius $A$ which is used as the parameter in both of the above expressions. An additional integral equation (solved numerically) is used in [45] to establish connection between the radius of the contact area and the radius of the cohesive zone. Further, the above approach was extended in [46] to a multilayered medium.

The above examples represent variety of approaches and mathematical models which cannot be exactly reduced to explicit $P-\delta$ functions. A the same time, they can be used in conjunction with the proposed extensions of the BG method.

\section{Appendix B. Substrate effect in DSI at small depth}

In the present work, a model of asymptotically thick layer is used in numerical simulations and the benchmarking of the BG method. This means that in the case of spherical indenter the smallness of the ratio of the contact radius to the coating thickness necessary leads to the requirement of small indentation depth. It is commonly assumed that at small indentation depth the substrate effect is negligible. However, it is not always so.

As Johnson ans Sridhar showed [49], the substrate effect during indentation of a coated medium can be taken into account by means of the introduction of dimensionless correction functions into the classical equations of the JKR theory. These correction functions show that the substrate effect depends on the interplay between the model geometry, and the elastic properties of both the coating and the substrate, which is not always straightforward.

Experimental studies demonstrate the substrate effect at small indentation depths. Consider, for example, the experiments on the indentation of coal samples by E. 
Kossovich et al [71]. In that work elastic moduli of coal were obtained by means of the Oliver-Pharr approach and nanoindentation with varying depth of maximum indentation $(150 \ldots 400 \mathrm{~nm})$ of a 14 micrometer films attached via a glue film to a rigid substrate. These moduli varied considerably depending on the maximum depth of indentation.

To assess the significance of the substrate effect numerically, in the present Appendix we use Finite Element Method (FEM) by means of ANSYS 18 Mechanical APDL (ANSYS, Inc., Canonsburg, PA, USA), and simulate non-adhesive indentation of a coated medium at small depth, using the values of material properties similar to those used in Section 4. Three cases were considered: (i) indentation of a half-space made entirely of the coating material (Material 1) using the analytical solution of the Hertz contact theory (the reference solution), (ii) indentation of a large finite specimen made entirely of the coating material (Material 1) modelled using FEM to validate the accuracy of the FEM model itself, (iii) indentation of a large finite specimen of the same size made of a coating (Material 1) and a stiffer substrate (Material 2), modelled in ANSYS as well.

FEM simulations were carried out in axisymmetric formulation. The finite-size specimen was modelled to have thickness $H_{F E M}=41 \mathrm{~mm}$, and radius $R_{F E M}=200$ $\mathrm{mm}$ (Figure 16a). When the coated medium was modelled, the coating thickness was set to $h=1 \mathrm{~mm}$, and the substrate thickness was set to $H_{F E M}-h=40 \mathrm{~mm}$. The coating elastic properties (Material 1) were set as $E_{1}=7.906 \mathrm{MPa}$ and $\nu_{1}=0.3$ (Young modulus and the Poisson's ratio correspondingly), the substrate elastic properties (Material 2) were $E_{2}=100 \mathrm{MPa}$ and $\nu_{2}=0.25$. The following element types were used: PLANE183 for modelling the substrate and the coating, TARGE169 and CONTA175 for modelling the contact pair. The indenter was assumed to be rigid.

The results shown in Figure 16b demonstrate that exact analytical Hertz solution (solid line) and the FEM solution for the large finite specimen (circles) have negligible difference. This confirms the accuracy of the FEM model.

Now consider the same FEM model with introduced substrate effect via the difference in the properties between Material 1, occupying the top $1 \mathrm{~mm}$ layer, and Material 2, occupying the rest of the model. The maximum simulated indentation depth was $1 \%$ of the coating thickness. The corresponding results (triangles in Fig. 16b) demonstrate difference up to $15 \%$ at indentation depth $1 \%$ of the coating thickness. Thus, the substrate effect is noticeable in this case. 


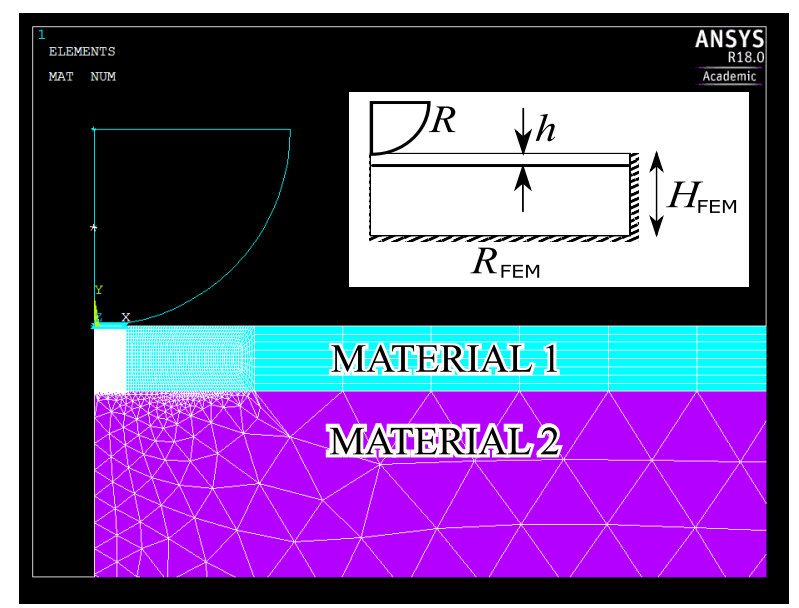

(a)

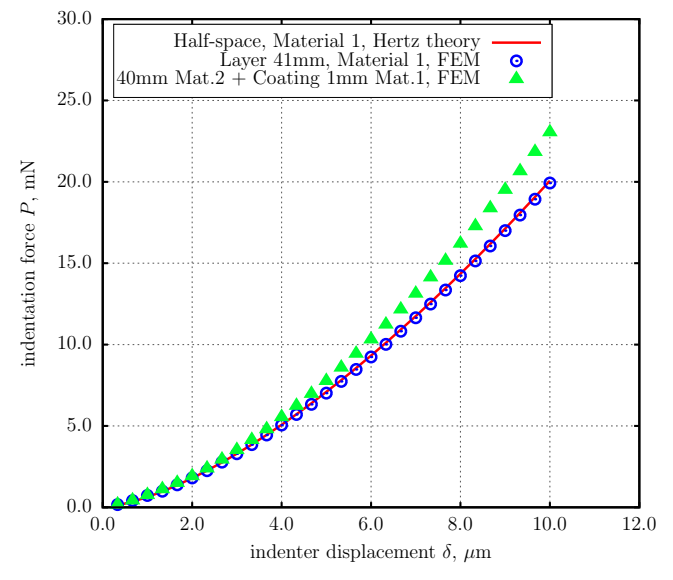

(b)

Figure 16: The substrate effect at small indentation depth: (a) the FEM model, (b) the comparison of force-displacement curves: the reference analytic Hertzian solution for a half-space (solid line), the FEM model without the substrate effect (circles), the FEM model with the substrate effect (triangles).

\section{Appendix C. Complete set of results obtained from the experimental DSI data by means of the extended BG method}

In this Appendix we present the complete set of results obtained by means of application of the extended two-stage BG method (17)-(18) with pre-fitting to the raw experimental data used earlier in the paper [14]. The details of the experiment and data processing is presented in the section 4.3 above.

In Figure 17-18 the complete set of results of identification of the properties of polyvinyl siloxane (PVS) is shown as the number of segments in the pre-fitting polygonal chain varies from 3 to 10 . The values of $E^{*}$ and $w$ were identified separately for each of the 24 available data sets. The result of each identification is represented as a dot in the figures. 


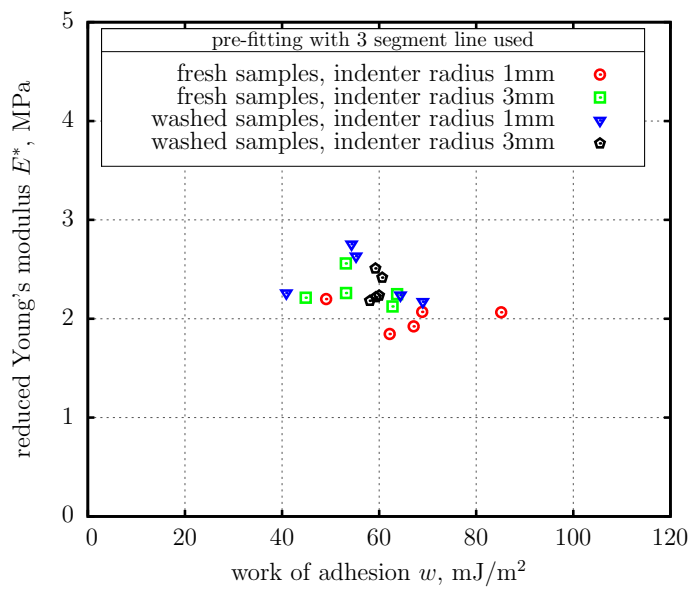

(a)

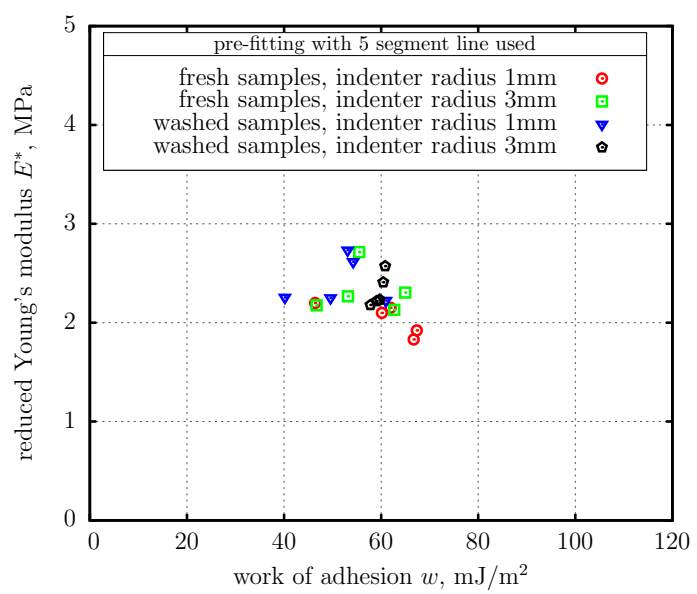

(c)

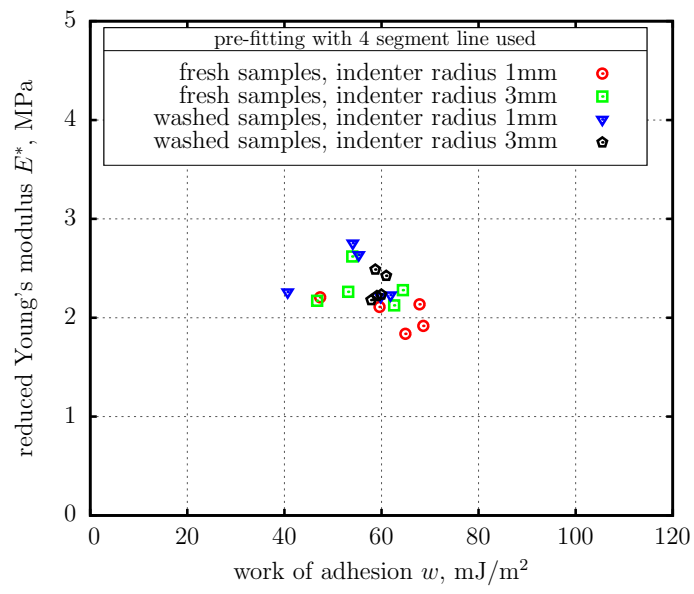

(b)

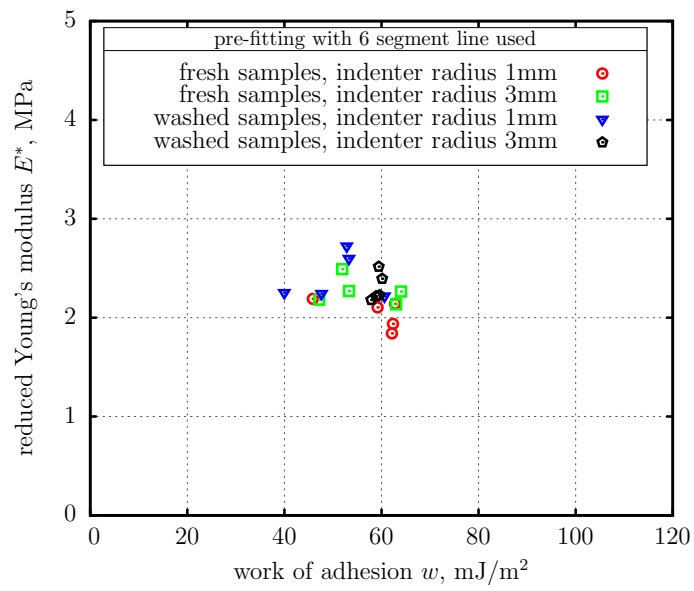

(d)

Figure 17: Material properties extracted using pre-fitting with polygonal chain. Number of segments in chain are correspondingly: 3 (a), 4 (b), 5 (c), 6 (d). 


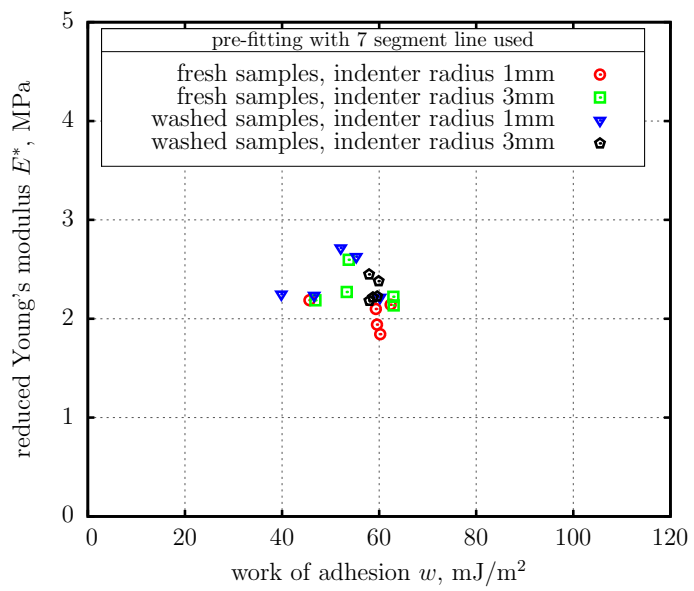

(a)

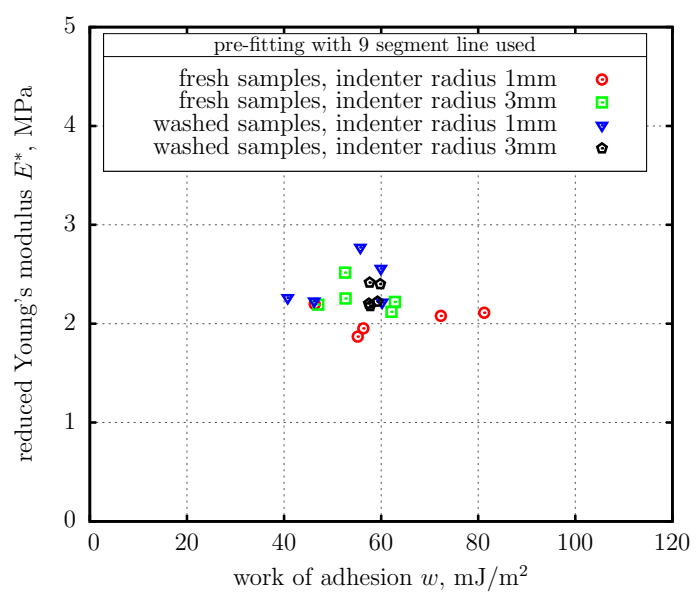

(c)

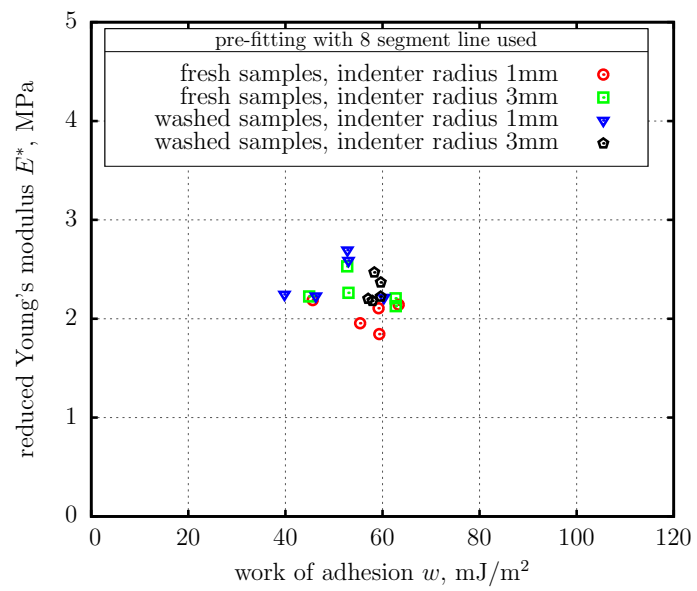

(b)

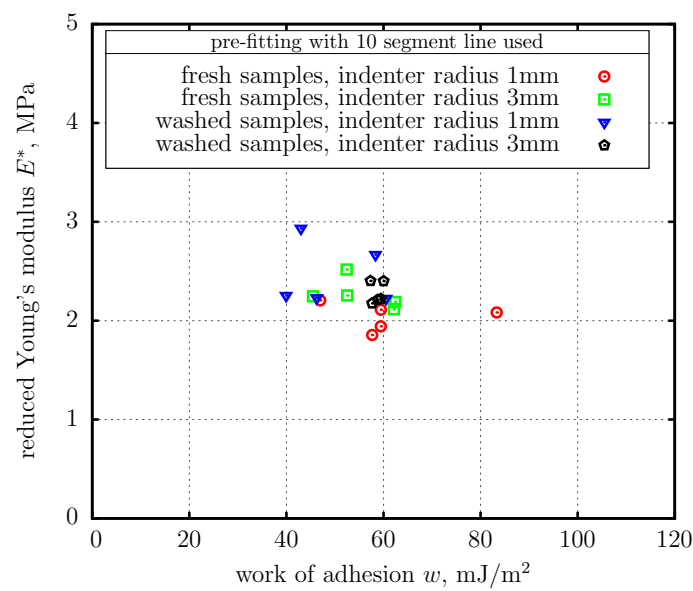

(d)

Figure 18: Material properties extracted using pre-fitting with polygonal chain. Number of segments in chain are correspondingly: 7 (a), 8 (b), 9 (c), 10 (d).

\section{References}

[1] Kendall, K. Molecular Adhesion and Its Applications; Kluwer Academic/Plenum Publishers: New York, 2001.

[2] Davis, J. R. Tensile Testing, 2nd.; ASM International: Materials Park, OH, 2004.

[3] Kendall, K. Thin-film peeling - the elastic term. J. Phys. D: Appl. Phys. 1975, $8,1449-1452$.

[4] Kalei, G. N. Some results of microhardness test using the depth of impression (in Russian). Mashinovedenie 1968, 4, 105-107.

[5] Wahl, K. J.; Asif, S. A. S.; Greenwood, J. A.; Johnson, K. L. Oscillating adhesive contacts between micron-scale tips and compliant polymers. J. Colloid Interface Sci. 2006, 296, 178-188.

[6] Ebenstein, D. M.; Wahl, K. J. A comparison of JKR-based methods to analyze quasi-static and dynamic indentation force curves. J. Colloid Interface Sci. 2006, 298, 652-662. 
[7] Borodich, F. M.; Keer, L. M. Evaluation of elastic modulus of materials by adhesive (no-slip) nanoindentation. Proc. R. Soc. London A 2004, 460, 507514.

[8] Borodich, F. M. The Hertz-type and adhesive contact problems for depthsensing indentation. Adv. App. Mech. 2014, 47, 225-366.

[9] Beach, E. R.; Tormoen, G. W.; Drelich, J.; Han, R. Pull-off force measurements between rough surfaces by atomic force microscopy. J. Colloid Interface Sci. 2002, 247, 84-99.

[10] Chaudhri, M. M.; Lim, Y. Y. Nanoindentation Techniques: A Critical Assessment of the Current Methods of Data Analysis. Key Engineering Materials 2007, 345-346, 1107-1114.

[11] Borodich, F. M.; Galanov, B. A. Non-direct estimations of adhesive and elastic properties of materials by depth-sensing indentation. Proc. R. Soc. London A 2008, 464, 2759-2776.

[12] Borodich, F. M.; Galanov, B. A.; Gorb, S. N.; Prostov, M. Y.; Prostov, Y. I.; Suarez-Alvarez, M. M. Evaluation of adhesive and elastic properties of materials by depth-sensing indentation of spheres. J. App. Phys. A: Mater. Sci. and Processing 2012, 108, 13-18.

[13] Borodich, F. M.; Galanov, B. A.; Gorb, S. N.; Prostov, M. Y.; Prostov, Y. I.; Suarez-Alvarez, M. M. An inverse problem for adhesive contact and non-direct evaluation of material properties for nanomechanics applications. Nanoscale Systems: Mathematical Modeling, Theory and Applications 2012, 1, 80-92.

[14] Borodich, F. M.; Galanov, B. A.; Gorb, S. N.; Prostov, M. Y.; Prostov, Y. I.; Suarez-Alvarez, M. M. Evaluation of Adhesive and Elastic Properties of Polymers by the BG Method. Macromol. React. Eng. 2013, 7, 555-563.

[15] Oliver, W. C.; Pharr, G. M. Improved technique for determining hardness and elastic modulus using load and displacement sensing indentation experiments. J. Mater. Research 1992, 7, 1564-1580.

[16] Ducker, W. A.; Senden, T. J.; Pashley, R. M. Direct measurement of colloidal forces using an atomic force microscope. Nature 1991, 353, 239-241.

[17] Butt, H.-J. Measuring electrostatic, van der Waals, and hydration forces in electrolyte solutions with an atomic force microscope. Biophys. J. 1991, 60, $1438-1444$.

[18] Kappl, M.; Butt, H.-J. The Colloidal Probe Technique and its Application to Adhesion Force Measurements. Particle 8 Particle Systems Characterization 2002, 19, 129-143.

[19] Makhlouf, A. Nanocoatings and Ultra-Thin Films. Technologies and Applications; Woodhead Publishing: Cambridge, 2011. 
[20] Perepelkin, N.; Kovalev, A.; Gorb, S.; Borodich, F. Estimation of the elastic modulus and the work of adhesion of soft materials using the extended Borodich-Galanov (BG) method and depth sensing indentation. Mech. Mat. 2019, 129, 198-213.

[21] Boggs, P. T.; Byrd, R. H.; Schnabel, R. B. A stable and efficient algorithm for nonlinear orthogonal distance regression. SIAM J. Sci. Stat. Comput. 1987, 8, $1052-1078$.

[22] Helfrich, H.-P.; Zwick, D. A trust region algorithm for parametric curve and surface fitting. J. Comput. App. Mathem. 1996, 73, 119-134.

[23] Atieg, A.; Watson, G. A. A class of methods for fitting a curve or surface to data by minimizing the sum of squares of orthogonal distances. J. Comput. App. Mathem. 2003, 158, 277-296.

[24] Ahn, S. J.; Rauh, W.; Cho, H. S.; Warnecke, H.-J. Orthogonal Distance Fitting of Implicit Curves and Surfaces. IEEE Trans. Pattern Analysis and Machine Intelligence 2002, 24, 620-638.

[25] Ahn, S. J. Least Squares Orthogonal Distance Fitting of Curves and Surfaces in Space; Springer-Verlag: Berlin, 2004.

[26] Ahn, S. J. Geometric Fitting of Parametric Curves and Surfaces. J. of Information Processing Systems 2008, 4, 153.

[27] Argatov, I. I.; Borodich, F. M.; Popov, V. L. JKR adhesive contact for a transversely isotropic layer of finite thickness. J. Phys. D: Appl. Phys. 2016, 49, 045307.

[28] Bulychev, S. I.; Alekhin, V. P.; Shorshorov, M. K.; Ternovskii, A. P.; Shnyrev, G. D. Determination of Young's modulus according to indentation diagram (in Russian). Industrial Laboratory 1975, 41, 1409-1412.

[29] Khrushchov, M. M.; Berkovich, E. S. Devices PMT-2 and PMT-3 for microhardness testing (in Russian); USSR Academy of Sciences Publising: Moscow, 1950.

[30] Giannakopoulos, A.; Suresh, S. Determination of elastoplastic properties by instrumented sharp indentation. Scripta Materialia 1999, 40, 1191 - 1198.

[31] ISO14577-1; International standard ISO 14577-1:2015. Metallic materials - Instrumented indentation test for hardness and materials parameters - Part 1: Test method; 2015.

[32] Galanov, B. A.; Dub, S. N. Critical comments to the Oliver-Pharr measurement technique of hardness and elastic modulus by instrumented indentations and refinement of its basic relations. J. Superhard Mater. 2017, 39, 373-389.

[33] Kossovich, E. L.; Borodich, F. M.; Epshtein, S. A.; Galanov, B. A.; Minin, M. G.; Prosina, V. A. Mechanical, structural and scaling properties of coals: depth-sensing indentation studies. App. Phys. A 2019, 125, 195. 
[34] Rundlöf, M.; Karlsson, M.; Wågberg, L.; Poptoshev, E.; Rutland, M.; Claesson, P. Application of the JKR Method to the Measurement of Adhesion to Langmuir-Blodgett Cellulose Surfaces. J. Colloid Interface Sci. 2000, 230, 441447.

[35] Carrillo, F.; Gupta, S.; Balooch, M.; Marshall, S.; Marshall, G.; Pruitt, L.; Puttlitz, C. Nanoindentation of polydimethylsiloxane elastomers: Effect of crosslinking, work of adhesion, and fluid environment on elastic modulus. $J$. Mater. Research 2005, 20, 2820-2830.

[36] Yu, Y.; Sanchez, D.; Lu, N. Work of adhesion/separation between soft elastomers of different mixing ratios. J. Mater. Research 2015, 30, 2702-2712.

[37] Johnson, K. L.; Kendall, K.; Roberts, A. D. Surface energy and the contact of elastic solids. Proc. R. Soc. London A 1971, 324, 301-313.

[38] Derjaguin, B. V.; Muller, V. M.; Toporov, Y. P. Effect of contact deformations on adhesion of particles. J. Colloid Interface Sci. 1975, 53, 314-326.

[39] Maugis, D. Contact, Adhesion and Rupture of Elastic Solids; Springer-Verlag: Berlin, 2000.

[40] Grierson, D. S.; Flater, E. E.; Carpick, R. W. Accounting for the JKR-DMT transition in adhesion and friction measurements with atomic force microscopy. Journal of Adhesion Science and Technology 2005, 19, 291-311.

[41] Kohn, J. C.; Ebenstein, D. M. Eliminating adhesion errors in nanoindentation of compliant polymers and hydrogels. Journal of the Mechanical Behavior of Biomedical Materials 2013, 20, 316 - 326.

[42] Sirghi, L.; Rossi, F. Adhesion and elasticity in nanoscale indentation. App. Phys. Lett. 2006, 89, 243118-243118.

[43] Borodich, F. M.; Galanov, B. A.; Perepelkin, N. V.; Prikazchikov, D. A. Adhesive contact problems for a thin elastic layer: Asymptotic analysis and the JKR theory. Mathematics and Mechanics of Solids 2019, 24, 1405-1424.

[44] Argatov, I. I.; Sabina, F. J. Asymptotic analysis of the substrate effect for an arbitrary indenter. The Quarterly Journal of Mechanics and Applied Mathematics 2013, 66, 75-95.

[45] Sergici, A. O.; Adams, G. G.; Müftü, S. Adhesion in the contact of a spherical indenter with a layered elastic half-space. J. Mech. Phys. Sol. 2006, 54, 18431861.

[46] Stan, G.; Adams, G. G. Adhesive contact between a rigid spherical indenter and an elastic multi-layer coated substrate. Int. J. Sol. Struct. 2016, 87, 1-10.

[47] Jin, F.; Guo, X.; Gao, H. Adhesive contact on power-law graded elastic solids: The JKR-DMT transition using a double-Hertz model. J. Mech. Phys. Sol. 2013, 61, 2473-2492. 
[48] Sridhar, I.; Johnson, K. L.; Fleck, N. A. Adhesion mechanics of the surface force apparatus. J. Phys. D: Appl. Phys. 1997, 30, 1710-1719.

[49] Johnson, K. L.; Sridhar, I. Adhesion between a spherical indenter and an elastic solid with a compliant elastic coating. J. Phys. D: Appl. Phys. 2001, 34, 683-689.

[50] Sridhar, I.; Zheng, Z. W.; Johnson, K. L. A detailed analysis of adhesion mechanics between a compliant elastic coating and a spherical probe. J. Phys. D: Appl. Phys. 2004, 37, 2886-2895.

[51] Domke, J.; Radmacher, M. Measuring the elastic properties of thin polymer films with the atomic microscope. Langmuir 1998, 14, 3320-3325.

[52] Dimitriadis, E. K.; Horkay, F.; Maresca, J.; Kachar, B.; Chadwick, R. S. Determination of elastic moduli of thin layers of soft material using the atomic force microscope. Biophysical J. 2002, 82, 2798-2810.

[53] Sirghi, L.; Ponti, J.; Broggi, F.; Rossi, F. Probing elasticity and adhesion of live cells by atomic force microscopy indentation. Eur. Biophys. J. 2008, 37, 935-945.

[54] Zhu, X.; Siamantouras, E.; Liu, K. K.; Liu, X. Determination of work of adhesion of biological cell under AFM bead indentation. J. Mech. Behavior Biomed. Mater. 2016, 56, 77-86.

[55] Bouchonville, N.; Meyer, M.; Gaude, C.; Gay, E.; Ratel, D.; Nicolas, A. AFM mapping of the elastic properties of brain tissue reveals $\mathrm{kPa} \mu \mathrm{m}(-1)$ gradients of rigidity. Soft Matter 2016, 12, 6232-6239.

[56] Notbohm, J.; Poon, B.; Ravichandran, G. Analysis of nanoindentation of soft materials with an atomic force microscope. J. Mater. Research 2012, 27, 229237.

[57] Lin, D. C.; Dimitriadis, E. K.; Horkay, F. Robust strategies for automated AFM force curve analysis-I. Non-adhesive indentation of soft, inhomogeneous materials. J. Biomech. Eng. 2007, 129, 430-440.

[58] Lin, D. C.; Dimitriadis, E. K.; Horkay, F. Robust strategies for automated AFM force curve analysis-II. Adhesion-influenced indentation of soft, elastic materials. J. Biomech. Eng. 2007, 129, 904-912.

[59] Wilks, S. S. Certain generalizations in the analysis of variance. Biometrika 1934, $24,471-494$.

[60] Wilks, S. S. Contributions to Probability and Statistics: Essays in Honor of Harold Hotelling; Olkin I.; Ghurye S.; Hoeffding W.; Madow W.; Mann H., Ed.; Stanford University Press, 1960; Chapter "Multidimensional statistical scatter", p 86-503.

[61] Tabor, D. Surface forces and surface interactions. J. Colloid Interface Sci. 1977, $58,2-13$. 
[62] Muller, V. M.; Yushchenko, V. S.; Derjaguin, B. V. On the influence of molecular forces on the deformation of an elastic sphere and its sticking to a rigid plane. J. Colloid Interface Sci. 1980, 77, 91-101.

[63] Borodich, F. M.; Galanov, B. A.; Suarez-Alvarez, M. M. The JKR-type adhesive contact problems for power-law shaped axisymmetric punches. J. Mech. Phys. Solids 2014, 68, 14-32.

[64] Johnson, K. L. Adhesion and friction between a smooth elastic spherical asperity and a plane surface. Proc. R. Soc. London A 1997, 453, 163-179.

[65] Alexandrov, V. M. On the solution of certain contact problems of the theory of elasticity. PMM J. Appl. Math. Mech. 1963, 27, 1490-1494.

[66] Alexandrov, V. M.; Vorovich, I. I. Contact problems for the elastic layer of small thickness. PMM J. Appl. Math. Mech. 1964, 28, 425-427.

[67] Argatov, I.; Mishuris, G. Contact Mechanics of Articular Cartilage Layers. Asymptotic Models; Springer International Publishing: Cham, Switzerland, 2015.

[68] Aghalovyan, L. Asymptotic theory of anisotropic plates and shells; World Scientific Publishing Co. Pte. Ltd.: Singapore, 2015.

[69] Argatov, I. I.; Mishuris, G. S.; Popov, V. L. Asymptotic modelling of the JKR adhesion contact for a thin elastic layer. The Quarterly Journal of Mechanics and Applied Mathematics 2016, 69, 161-179.

[70] Greenwood, J. A.; Johnson, K. L. An alternative to the Maugis model of adhesion between elastic spheres. J. Phys. D: App. Phys. 1998, 31, 3279-3290.

[71] Kossovich, E. L.; Borodich, F. M.; Bull, S. J.; Epshtein, S. A. Substrate effects and evaluation of elastic moduli of components of inhomogeneous films by nanoindentation. Thin Solid Films 2016, 619, 112-119. 